\title{
Felix Dirsch \\ Kulturrevolution oder Studentenbewegung? Ansätze zur Historisierung der Ereignisse von »1968«
}

\author{
1. Historisierung: Tragfähigkeit und Grenze \\ eines geschichtswissenschaftlichen Konzepts
}

Ereignisse von historischem Rang scheinen im Abstand von über einer Generation der Historisierung zu bedürfen. Vierzig Jahre nach dem Ende des »Dritten Reiches « setzte, im Anschluss an einen viel zitierten Aufsatz von Martin Broszat ${ }^{1}$, eine breite Debatte in der Geschichtswissenschaft², aber auch in der Öffentlichkeit, über

1 Martin Broszat, »Plädoyer für eine Historisierung des Nationalsozialismus «, in: ders., Nach Hitler. Der schwierige Umgang mit unserer Geschichte. Beiträge von Martin Broszat, hrsg. von Hermann Graml und Klaus-Dietmar Henke, München 1986, S. 159-173; nochmals aufgegriffen wurde die Thematik vom gleichen Autor in dem Beitrag: »Was heißt Historisierung des Nationalsozialismus? « in: Historische Zeitschrift 247 (1988), S. 1-14; zur Kritik an Broszats Perspektive vgl. Saul Friedländer: »Überlegungen zur Historisierung des Nationalsozialismus? « in: Dan Diner, (Hg.), Ist der Nationalsozialismus Geschichte? Zu Historisierung und Historikerstreit, Frankfurt a.M. 1987, S. 34-50; ein Austausch der Positionen beider Gelehrter findet sich in folgendem Beitrag: Martin Broszat / Saul Friedländer, »Um die >Historisierung< des Nationalsozialismus. Ein Briefwechsel « in: Vierteljahreshefte für Zeitgeschichte 36 (1988), S. 339-372.

2 Vgl. aus der Fülle an Stellungnahmen lediglich die ausführliche Zusammenfassung der unterschiedlichen Beiträge bei Uwe Backes / Eckhart Jesse / Rainer Zitelmann, »Was heißt >Historisierung< des Nationalsozialismus? « in: Uwe Backes / Eckhart Jesse / Rainer Zitelmann, (Hg.), Die Schatten der Vergangenheit. Impulse zur Historisierung des Nationalsozialismus, Frankfurt a.M./Berlin 1990, S. 25-57; zur Zurückweisung der Kritik an diesem Sammelband vgl. Eckhart Jesse, »Verfehlte Kritik an der >Historisierung< des Dritten Reiches. Eine Auseinandersetzung mit fragwürdigen Tendenzen bei Walter Hofer«, in: Mut. Forum für Kultur, Politik und Geschichte, Nr. 313 (September 1993), S. 51-57; zur Erklärung des Historisierungs-Theorems aus geschichtswissenschaftlicher Sicht vgl. Nicolas Berg, Der Holocaust und die westdeutschen Historiker. Erforschung und Erinnerung, 3., durchges. Auflage, Göttingen 2004, S. 35-46, hier S. 40, der Broszats Absicht als rationalen Versuch sieht, »Gedächtnis in Geschichte zu verwandeln«; statt vieler anderer Einwände vgl. die Kritik am Historisierungs-Postulat bei Hans Küng, Das Judentum, München 1991, S. 282 f., der sich keine Mühe macht, die Intentionen Broszats näher zu analysieren; besonders provokant wirkte auf Historiker-Zunft und Teile der Öffentlichkeit die Umsetzung des Historisierungs-Postulats bei Ernst Nolte. Er stellte die Frage nach Vorläufern hinsichtlich der Intention der Vergasung einer feindlichen Gruppe oder einer feindlichen Klasse. Zwar wurde dieser Wunsch gelegentlich geäußert, etwa bei den Anhängern Malthus' oder in der Phantasie einiger linker Schriftsteller wie Tucholsky. Nur: Es fehlten die Möglichkeiten der Realisierung des Beabsichtigten. So verbleiben solche Fälle extremer Polemik gegen den politischen 
die Notwendigkeit der Einordnung der NS-Herrschaft in das Kontinuum der deutschen Geschichte ein. Obwohl von allen Seiten eine gewisse Berechtigung dieses Anliegens konzediert wurde, auch von Kritikern des Unternehmens, überwog doch weithin die Skepsis. Die Kritiker wendeten ein, dass mit einem solchen Projekt zwangsläufig eine Relativierung des Nationalsozialismus verbunden sei. Das Ausmaß der Verbrechen verbiete jedoch die damit explizit oder implizit geforderte subjektive Distanz zum Analyseobjekt. Broszats Beitrag, erstmals 1985 im »Merkur « abgedruckt, sah in der Tat die Problematik. Er unterstrich, dass die nationalsozialistische Vergangenheit »durch neue weltgeschichtliche Gewalt- und Katastrophenerfahrungen an Singularität eingebüßt « habe. ${ }^{3}$ Dieser Satz erwies sich als Achillesferse seiner Argumentation. Die Gegner der Historisierung, die hierin eine Grenze der Methode erkannten, stießen sich zumeist an den Gefahren, welche aus der Nichtbeachtung eines Axioms herrühren nämlich der Unvergleichbarkeit der nationalsozialistischen Grausamkeiten, wobei selten die methodischen Probleme von »Singularität« berücksichtigt wurden ${ }^{4}$. Broszat konnte nicht ahnen, dass diese Redeweise seit den 1980er-Jahren in der politisch-journalistischen Alltagssprache einen außerordentlichen Aufschwung nehmen sollte. So lässt die öffentliche Debatte über die Singularität der NS-Untaten, die gewisse dogmatische und ausgrenzende Züge zeigte ${ }^{5}$, jenen Hiatus erkennen, den Broszat früh bemerkte: Er verwies auf die Unterscheidung von Moralität einerseits und historiographischem Verstehen andererseits. Ge-

Gegner im Bereich des Geistig-Ideellen. An der Verwerflichkeit dieser Projektionen besteht aber gleichwohl kein Zweifel; zur Argumentation vgl. Ernst Nolte, »Zwischen Geschichtslegende und Revisionismus? Das Dritte Reich im Blickwinkel des Jahres 1980«, in: »Historikerstreit«. Die Dokumentation der Kontroverse um die Einzigartigkeit der nationalsozialistischen Judenvernichtung, 8. Auflage, München/Zürich 1991, bes. S. 29 f.; vgl. zu einer groß angelegten, wenngleich umstrittenen Historisierung der distinkten "National-sozialismen ", in Europa Karlheinz Weißmann, Der Weg in den Abgrund. Deutschland unter Hitler 1933-1945 (Propyläen-Geschichte Deutschlands, Bd. 9), Berlin 1995; die heftige Kritik an der Studie steht im Widerspruch zur Leistung des Autors, wie auch erklärte Gegner (zumindest indirekt) einräumen mussten (vgl. dazu Ulrich Herbert, »Die sselbstbewußte Nation< und der Nationalsozialismus «, in: Die Zeit vom 1. Dezember 1995; vgl. auch die anerkennenden Worte in der Besprechung Klaus Hildebrands, in: Historische Zeitschrift, Bd. 264 [1997], S. 527-530); zur Einordnung des Historisierungs-Theorems in den Kampf um die zeithistorische Deutungshoheit vgl. Ulrich von Hehl, "Kampf um die Deutung. Der Nationalsozialismus zwischen >Vergangenheitsbewältigung , Historisierungspostulat und sneuer Unbefangenheit«", in: Historisches Jabrbuch 117 (1997), S. 406-436, bes. S. 429-431; 431-435.

3 Broszat, Plädoyer für eine Historisierung des Nationalsozialismus, aaO. (FN 1), S.159.

4 Vgl. dazu Hagen Schulze, »Fragen, die wir stellen müssen. Keine historische Haftung ohne nationale Identität«, in: »Historikerstreit«, aaO. (FN 2), S. 143-150, hier S. 144.

5 So rechtfertigte die CDU-Vorsitzende Angela Merkel 2003 den Fraktions- und Parteiausschluss des CDU-Abgeordneten Martin Hohmann vor allem damit, dass er die "Singularität des Holocausts « geleugnet habe. Es bleibt strittig, ob das der Fall war, zumal Hohmann bei seinem berüchtigten Tätervolkvergleich den coniunctivus irrealis verwendete und am Ende seiner Ausführungen explizit hervorhob, dass weder Deutsche noch Juden als Tätervolk bezeichnet werden können; ausführlich dazu: Fritz Schenk, Der Fall Hohmann ... und kein Ende. Mit dem Text des "Sondervotums" des Bundesparteigerichts der CDU, 2., erw. und überarbeitete Neuauflage, München 2005. 
schichtswissenschaftlich könne sehr wohl geboten sein, was unter »volkspädagogischen « Gesichtspunkten schädlich sein müsse. Ordne man die »fortschrittliche« Sozialpolitik des Nationalsozialismus in die Geschichte der Wohlfahrtsprogramme der europäischen Staaten ein, so seien über Deutschland hinausreichende Grundtendenzen zu erkennen. Der ehemalige Direktor des Münchner Instituts für Zeitgeschichte arbeitete, gestützt auf die Habilitationsschrift Marie-Luise Reckers, einen »Schub progressiver sozialpolitischer Neuerungen trotz der eher manipulativen und repressiven Funktionen der DAF « heraus. ${ }^{6}$ Das alles ist kaum zu leugnen, obwohl die Gefahren solcher Forschungsergebnisse auf der Hand liegen - zumal dann, wenn sie im parteipolitischen Kontext verkürzt propagiert werden.

Da die Diskussion über Nutzen und Nachteil der Historisierung bisher weitgehend auf die NS-Zeit beschränkt blieb, die sicherlich einen besonderen Stellenwert im »kollektiven Gedächtnis « der Nation einnimmt', ist im Hinblick auf eine fruchtbare Anwendung des Konzepts auf andere Zeiträume ganz allgemein zu fragen: Welche Vorteile bietet die Historisierung für die geschichtswissenschaftliche Betrachtung? Welche methodischen Gewinne lassen sich bei korrekter Anwendung daraus ziehen? Zunächst ist grundsätzlich festzustellen, dass »Historisierung « eine offenkundig vage und unpräzise Forderung darstellt. Sie braucht eine inhaltliche Konkretisierung. Auch Broszat kommt in seinem insgesamt programmatischen und weiterführenden Beitrag an einigen Stellen nicht über Andeutungen hinaus. Liest man seine Stellungnahme in toto, so wird aber seine Absicht klar: Broszat schreibt nicht als »Relativierer « - eine Bezeichnung, die in diffamierender Intention auf Ernst Nolte angewendet wurde -, sondern primär als linker Historiker. Das ist ersichtlich, wenn man die geschichtswissenschaftliche Richtung beachtet, gegen die er sich wendet. Broszat will zeigen, dass der Nationalsozialismus eben kein Dämon war, der 1933 auf der Bildfläche erschien und nach zwölf Jahren wieder unrühmlich von der geschichtlichen Bühne verschwand. Es gab vielfältige Strömungen, die der »deutsche Radikalfaschismus « (Ernst Nolte) in äußerst destruktiver Weise instrumentalisierte, die aber bereits lange vor seiner Machtergreifung virulent waren, an primärer Stelle der Antisemitismus. Ebenso brachte der Nationalsozialismus vereinzelt Impulse hervor, die ihn überlebten und unter völlig anderen Vorzeichen in seinen beiden Nachfolgestaaten, Bundesrepublik und DDR, weiter existierten. Erwähnt wurde diesbezüglich bereits die Sozialpolitik. Die »Dämonologik « (Dolf Sternberger) als Deutungskonzept des »deutschen Faschismus«, seine Einordnung

6 Broszat, Plädoyer für eine Historisierung des Nationalsozialismus, aaO. (FN 1), S. 171 f.; mit anderem »erkenntnisleitenden Interesse « hat Götz Aly, Hitlers Volksstaat. Raub, Rassenkrieg und nationaler Sozialismus, durchges. und erw. Ausgabe, Frankfurt a.M. 2006, das schwierige Thema des »nationalen Sozialismus « bzw. des nationalsozialistischen Wohlfahrtsstaates wieder aufgegriffen. Anders als Reckers akademische Abhandlung schaffte die Studie Alys kurz nach ihrem Erscheinen den Sprung in die »Tagesthemen «, was für eine historiographische Darstellung, auch wenn sie stark populärwissenschaftlich ausgerichtet ist, eher außergewöhnlich ist.

7 Zur Vielfalt der individuellen wie kollektiven Konstruktionen der Vergangenheit vgl. aus der im letzten Jahrzehnt deutlich angewachsenen Literatur zu dieser Thematik Aleida Assmann, Der lange Schatten der Vergangenheit. Erinnerungskultur und Geschichtspolitik, München 2006. 
als »Betriebsunfall« liegt dem heutigen Betrachter der jüngeren deutschen Geschichte meist so fern wie nur möglich. Broszat weiß jedoch, dass derartige Erklärungsmuster in der konservativen Historiographie nach 1945 weit verbreitet waren. ${ }^{8}$ Seine Kritik an der liberal-konservativen Geschichtsschreibung wird auch an der Ablehnung bloßer moralisierender Gesinnungsappelle und meist fruchtloser Versuche der Pauschaldistanzierung deutlich, wie sie beispielsweise in Golo Manns viel gelesener deutscher Geschichte des 19. und 20. Jahrhunderts (1958 erstmals veröffentlicht) zum Vorschein kommen. Der Sohn des berühmten Schriftstellers macht an vielen Stellen des Werks keinen Hehl daraus, wie sehr er Hitler verachtet, der überall als »Scheusal« dargestellt wird. Er präsentiert ihn verstümmelt als »H.«. Nun ist das nach Broszat aber kein Zeichen für seriöse Historie, denn solche Werturteile sind eigentlich trivial. Mann publizierte als Zeitgenosse des Regimes, so dass er auf diese Weise seinen Status als Opfer nochmals reflektierte. 1985 war aber ein Großteil der unmittelbaren Zeitgenossen bereits verstorben, und die unausweichliche Fortsetzung dieses Trends hatte nachhaltige Auswirkungen auf die Geschichtsschreibung9. Im Hinblick auf die Erlebnisgeneration ist bei einem solchen Umgang mit der Vergangenheit eine Gefahr evident: Pauschaldistanzierungen können entlastend wirken und entheben öfters von der genauen Untersuchung des individuellen Schuldanteils.

Bezüglich einer neuen Anwendung des Historisierungs-Konzepts ist Folgendes festzuhalten: Historische Ereignisse von erheblicher Relevanz, gerade für die Nachwelt, verlieren durch eine konsequente Einordnung in die Geschichte jedweden mythologischen oder dämonischen Hintergrund. Gerade die Geschichtsschreibung über den Nationalsozialismus war aus verschiedenen und sehr verständlichen Gründen häufig in Gefahr, Ergebnisse mythisch oder dämonisch zu verbrämen - nicht zuletzt deshalb, um auf den besonderen Stellenwert der Auswirkungen des Regimes für die unmittelbare Gegenwart hinzuweisen. ${ }^{10}$ Worauf angesehene wie umstrittene Historiker (von Jan Assmann bis Ernst Nolte) hinwiesen - das wurde - mit einem time-lag von einigen Jahren - auch von Politikern rezipiert, die Auschwitz als »Gründungsmythos der Bundesrepublik Deutschland« (Joseph Fischer) betrachten.

8 Vgl. von Hehl, Kampf um die Deutung. Der Nationalsozialismus zwischen »Vergangenheitsbewältigung «, Historisierungspostulat und »neuer Unbefangenheit«, aaO. (FN 2), S. 410 f.; Broszat / Friedländer, Um die >Historisierung des Nationalsozialismus. Ein Briefwechsel, aaO. (FN 1), S. 365; Hans Mommsen, »Neues Geschichtsbewußtsein und Relativierung des Nationalsozialismus«, in: »Historikerstreit«, aaO. (FN 2), S. 156-173, hier S. 174, der die Deutung des Nationalsozialismus als »singulären Einbruch in die Kontinuität der deutschen Geschichte« i. S. einer ursprünglichen »Schlachtordnung konservativen Denkens« sieht, die angeblich erst in den 1980er-Jahren aufgegeben worden sei; grundlegend für die Beschäftigung mit dem Verhältnis von Historie und Holocaust in den 1950er- und 1960er-Jahren Berg, Der Holocaust und die westdeutschen Historiker. Erforschung und Erinnerung, aaO. (FN 2); zu dieser viel diskutierten Studie vgl. Felix Dirsch, in: Religion-Staat-Gesellschaft. Zeitschrift für Glaubensformen und Weltanschaunngen 7 (2006, Heft 1), S. 121-124.

9 Die Zeitgeschichtsforschung hat diesen Trend unter der Überschrift »Abschied von der Zeitzeugenschaft« abgehandelt; vgl. dazu Norbert Frei, »Abschied von der Zeitzeugenschaft. Der Nationalsozialismus und seine Erforschung auf dem Weg in die Geschichte«, in: ders., 1945 und wir. Das Dritte Reich im Bewußtstein der Deutschen, München 2005, S. 41-62.

10 Vgl. dazu das Urteil von Jan Assmann, Das kulturelle Gedächtnis. Schrift, Erinnerung und politische Identität, zweite, durchges. Auflage, München 1997, S. 76, der den »Holocaust« zur »fundierenden Geschichte und damit zum Mythos geworden« sieht. 
Auf die schillernde Ambivalenz, die der Begriff impliziert, kann an dieser Stelle nicht eingegangen werden. Es soll lediglich erwähnt werden, dass berüchtigte Vertreter des Revisionismus mit dem Begriff ebenso hantieren wie diejenigen ${ }^{11}$, die die dauerhafte Bedeutung der Judenvernichtung als bleibende Aufgabe einer universalen, weltweiten Humanisierung auch für die Gegenwart hervorheben - ganz in der Nachfolge Theodor W. Adornos, der »nach Auschwitz « einen neuen kategorischen Imperativ für die Menschheit gegeben sah: Solches dürfe nie wieder passieren!

\section{Zur Möglichkeit und Notwendigkeit der Übertragung des Historisierungs-Konzepts auf »1968«}

Als Broszat seinen grundlegenden Aufsatz konzipierte, waren die Vorbereitungen auf die Gedenktage zum vierzigsten Jahrestag der Kapitulation des deutschen Reiches in vollem Gange. Der gleiche Zeitraum trennt uns heute von den heterogenen Ereignissen, die man üblicherweise mit der schillernden Jahreszahl »1968 « verbindet. Es ist einsichtig, dass chronologische Abstände, für sich genommen, noch kein Argument bilden. Was mentale wie zeitgeschichtliche Zäsuren anbetrifft, stellt man »1945« - trotz der vielfältigen Differenzen - gern mit »1968« und »1989« in eine Reihe. Betrachtet man jedoch die »Zeitgeschichte als Streitgeschichte « ${ }^{12}$, so fällt Folgendes auf: Die großen Kontroversen dieser Disziplin, die auch die Öffentlichkeit aufwühlten, drehen sich alle um die Zeit von 1933-1945. Das gilt sogar für die Auseinandersetzung um die Thesen von Fritz Fischer, die - auf den ersten Blick betrachtet - eigentlich die Debatte von 1914 und die Zeit davor berühren. ${ }^{13}$ Letztlich geht es aber bei dieser historiographischen Diskussion in den frühen 1960erJahren um die Kontinuität nationalistischer, antidemokratischer und antisemitischer Eliten. Es fällt auf, dass der von Fritz Fischer betriebene Zerfall der »nationalen Meistererzählung «(Jarausch) zu einem Zeitpunkt stattfand, als sich viele politische, soziale und kulturelle Faktoren bündelten, nämlich in dem relativ kurzen Zeitraum von 1959-1962, der später als »Schwellenperiode « im Hinblick auf »1968 « begriffen wurde. ${ }^{14}$ Doch nicht nur die große Historikerdebatte um die Bedeutung des Ersten

11 Dezidiert kritisch gegenüber dem Revisionismus: Deborah E. Lipstadt, Betrifft: Lengnen des Holocaust, Zürich 1994.

12 Martin Sabrow / Ralph Jessen / Klaus Große Kracht, (Hg.), Zeitgeschichte als Streitgeschichte. Grosse Kontroversen seit 1945, München 2003.

13 Zur Relevanz der Fischer-Kontroverse für die Geschichtswissenschaft vgl. Konrad H. Jarausch, »Der nationale Tabubruch. Wissenschaft, Öffentlichkeit und Politik in der Fischer-Kontroverse" und Imanuel Geiss, »Zur Fischer-Kontroverse - 40 Jahre danach «, aaO. (FN 12), S. 20-40 u. S. 41-57.

14 Zum »Schwellenjahr 1960«, das erhebliche Auswirkungen für die Kultur der Bundesrepublik hatte, vgl. die kurze Zusammenfassung bei Felix Dirsch, "Mit spitzer Feder gegen den Zeitgeist. Ausgewählte konservative Zeitschriften und ihre Kritik an kulturrevolutionären Tendenzen", in: Hartmuth Becker / Felix Dirsch / Stefan Winckler, (Hg.), Die 68er und ibre Gegner. Der Widerstand gegen die Kulturrevolution, 2. Auflage, Graz 2004, S. 64-71 (dort auch Hinweise auf weitere der zahlreichen Belegstellen in der Literatur); vgl. zum »Paradigmenwechsel des Jahres 1960 « auch Caspar von Schrenck-Notzing, Charakterwäsche. Die Re-education der Deutschen und ibre bleibenden Auswirkungen, erw. Neuausgabe, 2. Auflage, Graz 2005, S. 10 f. 
Weltkrieges und seiner Folgen, auch die - wenngleich eher spärlichen - Kontroversen in der Geschichtsschreibung um $\gg 1968 \ll^{15}$ oder um das gleichfalls weltgeschichtlich epochale Datum von »1989 «16 - all das lässt sich auf die Phase zwischen 1933 und 1945 hin fokussieren. Das Aufkommen der 68er-Kulturrevolte kann man als Auseinandersetzung mit der belasteten Vätergeneration deuten. Daran ändert auch die Tatsache nichts, dass die Ursachen dieser Bewegung heute erheblich vielschichtiger gesehen werden. Die bedeutsamen Umbrüche um 1990 herum werden gemeinhin als Überwindung einiger wesentlicher Folgen des Weltkriegsendes interpretiert.

Wie ist nun die Forderung nach einer Historisierung der 68er-Bewegung zu begründen? Welche Aspekte hilft dieses geschichtswissenschaftliche Konzept zu klären? Obwohl die von Zeitzeugen gelegentlich angestellten Parallelen zwischen »1933« und »1968« nur von untergeordnetem Wert sind und weitgehend vernachlässigt werden können ${ }^{17}$, verbindet die Betrachtung der Periode von 1933-1945 und der Blick auf die Ereignisse der späten 1960er-Jahre in der historiographischen wie tagespolitischen Aufarbeitung eine nicht selten stark moralisch ausgerichtete Wahrnehmung und Wertung. Aus der Retrospektive will eine Interpretationsrichtung mit Zähnen und Klauen die Errungenschaften des großen kulturrevolutionären Um-

15 Vgl. etwa die Diskussion um den angeblichen oder tatsächlichen Antisemitismus einiger 68er, im Anschluss an die entlarvenden Stellungnahmen Dieter Kunzelmanns; dazu Wolfgang Kraushaar, Die Bombe im Jüdischen Gemeindehaus, Hamburg 2005.

16 Vgl. zu den Diskussionen um »1989 « Martin Sabrow, »Die Historikerdebatte über den Umbruch von 1989«, aaO. (FN 12), S. 114-137.

17 Diese Parallelen verfolgten jedoch nicht nur denunziatorisch-polemische Ziele, denn einzelne Gemeinsamkeiten sind durchaus nicht von der Hand zu weisen; Joachim C. Fest, Ich nicht. Erinnerungen an eine Kindheit und Jugend, 2. Auflage, Berlin 2006, S. 363, betont die Gefahren, die daraus entstanden, dass eine »politisch wirre und moralisch großtuerische Jugend die Welt aus einem Punkt « erklären wollte, obwohl die Folgen einer solchen Auffassung nur wenige Jahrzehnte vorher »offenbar « wurden; der Sozialdemokrat Richard Löwenthal, Der romantische Rückfall, Stuttgart 1970, S. 13, zeigt einige Affinitäten zwischen den »Ideen des Nationalsozialismus « und seinen heftigen Kritikern auf: »Die heutige Radikalisierung im Engagement der linksstehenden Intellektuellen ist somit nicht nur bewußter Gegenschlag gegen die Ideen des Nationalsozialismus und die Schrecken seiner Herrschaft, die den Kritikern wie uns allen noch in den Knochen stecken. Sie ist auch unbewußte Fortsetzung einiger der geistigen Strömungen, die diese Schrecken mit ermöglicht haben«; Hermann Lübbe, »Konzessionismus dient aber nie der Beschwichtigung, sondern er hat Selbstschädigung zur Konsequenz«, aaO. (FN 14), S. 37-63, hier S. 49 f., zitiert eine jüdische Remigrantin, die beim Anblick der "Studenten unter wallenden Fahnen und Führerbildern im Laufschritt in der Heidelberger Hauptstraße« in Tränen ausbrach und befand: »Jetzt fängt das wieder an«; der vom Judentum zur katholischen Kirche konvertierte Philosoph Helmut Kuhn, Die Kirche im Zeitalter der Kulturrevolution (Herkunft und Zukunft, Bd. 6), Graz/Wien/Köln 1985, S. 152 f., sieht besondere Parallelen zwischen der ersten deutschen Jugendbewegung, nach dem Ersten Weltkrieg, und der "zweiten deutschen Jugendbewegung « (Hermann Lübbe), die bekanntlich öfters skandierte: »Trau keinem über dreißig! «. Bei linken Nationalsozialisten wie den Gebrüdern Strasser, die Hitler später aus der Partei drängte, hieß das noch in den 1920er-Jahren: »Macht Platz ihr Alten! « Weitaus trivialer mutet der Vergleich bei Ludolf Hermann, »Hitler, Bonn und die Wende. Wie die Bundesrepublik ihre Lebenskraft zurückgewinnen kann«, in: Die politische Meinung 28 (1983) Nr. 204, S. 1315, an, der das » Trauma Hitler« neben das »Trauma 1968«stellt. 
bruchs verteidigen ${ }^{18}$, eine andere konstatiert hingegen negative »Spätfolgen der Kulturrevolution « ${ }^{19}$. Von der Annahme einer »Fundamentalliberalisierung « (Habermas) bis zu »Endstation Terror« (Lübbe) reicht das Interpretationsspektrum der Umbrüche. Beide Perzeptionsweisen stellen die - häufig nur vermuteten - Konsequenzen der geschichtlichen Ereignisse in den Mittelpunkt, nicht die Vorfälle selbst. Das bringt kaum zu übersehende Deutungsschwierigkeiten mit sich: Tendenzen von säkularem Rang, etwa die Bedeutungsminderung von Ehe und Familie oder die Zunahme von individualistisch-hedonistischen Verhaltensweisen, lassen sich kaum monokausal erklären. ${ }^{20}$ Die Relation von Ursache und Wirkung ist in diesem Kontext, wie auch in anderen historischen Zusammenhängen, schwer aufzuhellen. Dar-

18 Exemplarisch seien genannt: Jürgen Habermas, Protestbewegung und Hochschulreform, Frankfurt a.M. 1969; Oskar Negt, Achtundsechzig. Politische Intellektuelle und die Macht, Göttingen 1995; Uwe Wesel, Die verspielte Revolution. 1968 und die Folgen, München 2002.

19 68er-Kritik findet sich in den meisten Beiträgen der folgenden Sammelbände: Franz Schneider, (Hg.), Dienstjubiläum einer Revolte. »1968« und 25 Jahre, München 1993 und Venanz Schubert, (Hg.), 1968: 30 Jahre danach (Wissenschaft und Philosophie, Bd. 17), St. Ottilien 1999; von den wichtigen Gegnern der Kulturrevolution sind auswahlweise anzuführen: Günter Rohrmoser, Geistiges Vakuum - Spätfolgen der Kulturrevolution. Plädoyer für die christliche Vernunft, Bietingheim/Baden 1997; Hermann Lübbe, Unsere stille Kulturrevolution, Zürich 1976; ders., Endstation Terror. Rückblick auf lange Märsche, Stuttgart 1978; Robert Spaemann, Zur Kritik der politischen Utopie. 10 Kapitel politischer Philosophie, Stuttgart 1977; zu Lübbes und Spaemanns Kritik an einigen verfassungsdelegitimierenden Postulaten der »Neuen Linken« vgl. Ulrich Zellenberg, »Verfassungsstaat und Wahrheit. Zur Aktualität neokonservativer Argumente zur Verteidigung der liberalen Demokratie «, aaO. (FN 14), S. 12-36; kürzlich wurden die gemeinsamen philosophischen Hintergründe der genannten 68er-Gegner herausgearbeitet, die nicht zufällig aus der gleichen akademischen Schule stammen: Jens Hacke, Philosophie der Bürgerlichkeit. Die liberalkonservative Begründung der Bundesrepublik, Göttingen 2006, wobei der Verfasser rechtskonservativ einzuordnende Ritter-Schüler wie Günter Rohrmoser, Bernard Willms oder Reinhart Maurer fast vollständig aus seinen Erörterungen ausklammert. Hinweise zu dieser Studie finden sich u.a. in der Besprechung von Felix Dirsch, in: Zeitschrift für Politik 54 (2007), S. 107 f.; aus der anwachsenden 68er-Kritik im Rahmen der belletristischen Literatur vgl. Uwe Tellkamp, Der Eisvogel, Reinbek b. Hamburg 2006, wo die Bezeichnung »Morbus 68 « vorkommt; Sophie Dannenberg, Das bleiche Herz der Revolution, Berlin 2006, schreibt ihren Frust als Tochter eines 68er-Paares nieder. Sie hat als Kind von Kulturrevolutionären Schäden insbesondere durch die antiautoritäre Erziehung erlitten und reflektiert nunmehr die Folgen als Frau im mittleren Lebensalter.

20 Rohrmoser, der gelegentlich zu einer solchen Verkürzung zu neigen scheint, hat das durchaus richtig gesehen, wenn er schreibt: »Natürlich sind andere Faktoren und Bedingungen [als die familienkritischen bis -zerstörenden Einflüsse der Lehre der Frankfurter Schule, F.D.] hinzugekommen, aber das gehört auch mit zum Resultat (Günter Rohrmoser, »Brauchen wir eine Kulturrevolution? «, in: Förderstiftung Konservative Bildung und Forschung [FKBF]: Unsere Agenda 6 [2007], S. 10); in der quaestio facti - mit den Worten Kants gesprochen - stimmt mit Rohrmoser auch Wolfgang Kraushaar überein, wenn er in einem Interview feststellte: »Ich will diese Verdienste gar nicht pauschal bestreiten, begreife das Erbe dieser Zeit jedoch weniger kausallogisch« (» Wie wir uns befreiten. Michael Naumann und Wolfgang Kraushaar im Gespräch über 1968, die Folgen und das Selbstverständnis der Republik«. in: DIE ZEIT - Geschichte 2 [2007], S. 32-37, hier S. 34). 
über hinaus ist ein weiteres Problem zu konstatieren. Als der ehemalige Außenminister Joseph Fischer 2001 mit den Ereignissen von »1968 « konfrontiert wurde, was sowieso differenzierungsbedürftig ist ${ }^{21}$, griffen ihn journalistische wie politische Gegner mehr als - nach Umfragen hin - beliebten Staatsmann der unmittelbaren Gegenwart als einen in die damaligen Ereignisse Involvierten an. So kann man an diesem Beispiel ein durchaus weiter verbreitetes Phänomen erkennen: die Instrumentalisierung der zeitgeschichtlichen Vergangenheit für politische Positionen der unmittelbaren Gegenwart. Ingrid Gilcher-Holtey hat diese Diskrepanz wohl im Blick, wenn sie davon spricht, dass es zwar eine »Vielzahl von Thesen über >1968« und eine mannigfache Zahl von Folgenabschätzungsversuchen gebe, dennoch aber ein Mangel an Monographien über die Bundesrepublik im gleichen Jahr festzustellen sei, weiterhin existierten zahlreiche Desiderate auf einzelnen Themenfeldern, beispielsweise der Bedeutung der NS-Problematik hinsichtlich der Mobilisierung der Studenten. ${ }^{22}$

Es erscheint evident, dass es der Klärung historischer Fakten nicht dienlich ist, wenn man sie primär aus der Perspektive ihrer - tatsächlichen oder vermuteten Folgen her deutet. Konkret auf 1968 « angewendet heißt das: In der Rückschau wurde der Protest des Zeitraums um 1970 herum zumeist als Anstoß zu geistigen, kulturellen und gesellschaftlichen Veränderungen interpretiert. Es kam - so eine immer wiederkehrende Erklärung - zu vielfältigen Formen der Modernisierung: Von einer neuen Art des Umgangs mit der Vergangenheit bis hin zur angeblich nunmehr erst irreversiblen Verwestlichung reicht üblicherweise das breite Spektrum der von der Forschung herausgearbeiteten und von Zeitgenossen bestätigten Folgewirkungen. Wenn man sich jedoch die häufig wiederholten Ziele der APO-Führer, von Rudi Dutschke über Bernd Rabehl und Horst Mahler bis zu Hans-Jürgen Krahl, verdeutlicht, wird klar, dass nicht wenige dieser Vorstellungen mit den faktischen oder auch nur vermuteten Konsequenzen in einem Spannungsverhältnis stehen. Der Antiautoritäre Hans-Jürgen Krahl, um exemplarisch zu argumentieren, propagierte keine »Fundamentaldemokratisierung " (Karl Mannheim), sondern wollte der »bbürgerlichen Wissenschaft den Garaus machen ${ }^{23}$ Die marxistische Ausrichtung

21 Joschka Fischer trat als politischer Akteur in den späten 1960er-Jahren, was aus Altersgründen verständlich ist, kaum hervor, sieht man von einem eher mysteriösen Kongress der damals eindeutig terroristischen PLO einmal ab. Fischer engagierte sich weitaus stärker in den 1970er-Jahren, als die APO längst zerfallen war und sich mehr oder minder radikale Gruppen gebildet hatten, die vielen Aktivisten Unterschlupf boten. $\mathrm{Zu}$ Fischers Frankfurter Aktivitäten vgl. lediglich Christian Schmidt, »Wir sind die Wabnsinnigen...". Joschka Fischer und seine Frankfurter Gang, München/Düsseldorf 1999.

22 Ingrid Gilcher-Holtey, »>1968<- Eine versäumte Kontroverse«, aaO. (FN 12), S. 58-73.

23 Günter C. Behrmann, »Kulturrevolution. Zwei Monate im Sommer 1967 «, in: Clemens Albrecht / Günter C. Behrmann / Michael Bock / Harald Homann / Friedrich H. Tenbruck, Die intellektuelle Gründung der Bundesrepublik. Eine Wirkungsgeschichte der Frankfurter Schule, Frankfurt a.M./New-York 2000, S. 312-386, hier S. 344; zur kämpferischen Haltung Krahls vgl. dessen Vorstellungen im Rahmen eines aufgezeichneten Gerichtsplädoyers: Hans-Jürgen Krahl, »Angaben zur Person«, in: 1968. Eine Enzyklopädie, zusammengestellt von Rudolf Sievers, Frankfurt a.M., S. 331-343, hier S. 341, der den Kampf als Einlösung des Emanzipationsversprechens propagiert. 
mit allen ihren Postulaten, etwa den ökonomischen, die heute weitgehend nur noch Kopfschütteln hervorrufen, gilt in der Retrospektive als klarstes Zeichen von Weltfremdheit. Es reicht freilich nicht, nur zwischen den Zielen der 68er-Bewegung und den soziokulturellen Folgen zu unterscheiden. Nur eine nachhaltige Historisierung kann die Hintergründe dieser Differenzierung aufhellen, die selbst aber wiederum begründungsbedürftig ist.

Ein zweites Argument für die Notwendigkeit der Historisierung der »Kulturrevolution « - auch die Tragfähigkeit dieses Ausdrucks bleibt in der politologischen Forschung umstritten ${ }^{24}$ - ist an dieser Stelle noch anzudeuten. Wie die Periode von 19331945 ist »1968 « nicht nur moralisierungs-, sondern auch mythenanfällig. ${ }^{25}$ Eine derartige Tendenz muss seriöser zeitgeschichtlicher Forschung grundsätzlich ein Dorn im Auge sein, unabhängig davon, welche historische Periode konkret behandelt wird. Im Anschluss an das Ende der nationalsozialistischen Gewaltherrschaft am 8. Mai 1945 bildete sich im Westen Europas ein umfassender Freiheitsmythos heraus. Ursprünglich waren Mythen Wissensspeicher in schriftlosen Kulturen. Obwohl für eine derartige Funktion schon lange kein Bedarf mehr vorhanden ist, halten sich viele Mythen tapfer bis in die Gegenwart. Aus ihrer sinnstiftenden Funktion heraus gewinnen sie Bedeutung für das Existenzverständnis des Menschen auch heute; sie sind somit stets Ausdruck einer Weltanschauung. Mythen zielen häufig auf eine Aufhellung der menschlichen Ursprünge: Woher komme ich? Wo liegt der Anfang meiner Gemeinschaft? 1945 ist ein solches Gründungsdatum. Ab der so symbolträchtigen »Stunde Null«, die es bekanntlich nie gab, soll alles besser geworden sein.

24 Wolfgang Weber, »Die >Kulturrevolution< 1968 « in: Volker Dotterweich, (Hg.), Kontroversen in der Zeitgeschichte. Historisch-politische Themen im Meinungsstreit, München 1998, S. 207-228; zur Präzisierung dessen, was unter »Kulturrevolution « gemeint war, vgl. auch Behrmann, Kulturrevolution. Zwei Monate im Sommer 1967, aaO. (FN 23); um eine genauere Definition des facettenreichen Terminus »Kulturrevolution « bemüht sich Günter Rohrmoser, Der Ernstfall. Die Krise unserer liberalen Republik, Berlin/ Frankfurt a.M. 1994, S. 321: »Unter Kulturrevolution verstehe ich den über die Institutionen der Bewußtseinsbildung, also über den Kindergarten, Schule, Universität, öffentliche Meinung, Kirchen, Theater und Kunst, laufenden Prozeß, den Menschen mit Hilfe der Sprache in seinem Bewußtsein und seinen Bedürfnissen so zu verändern, daß er nur Normen und Werten zustimmt, die erst in einer anderen - nämlich einer zukünftigen sozialistischen - Gesellschaft verwirktlicht werden sollten... Er [der kulturrevolutionäre Prozeß] wirkt revolutionierend, wenn er in den Dienst der Aufgabe gestellt wird, überkommene Werte und Orientierungen aufzubauen, sie ideologiekritisch aufzulösen und an ihre Stelle neue Werte, neue Normen, neue Orientierungen, neue Formen der Sinngebung zu setzen «.

25 Diese Moralisierungsanfälligkeit erkennt man vor allem daran, wenn als Resümee über »1968 « nicht selten konstatiert wird, es sei nicht alles schlecht bzw. gut gewesen, was an »Errungenschaften « hervorgebracht wurde. Solche Feststellungen dürfen als trivial gelten (vgl. als eines der zahlreichen journalistischen Beispiele dafür: Titelthema Der Spiegel vom 29.10.2007, »Es war nicht alles schlecht! Gnade für die 68er «; als Variante des mittlerweile in manchen Kreisen populären 68er-Bashings darf der Bestseller des Chefredakteurs der Bild-Zeitung, Kai Diekmann, Der große Selbstbetrug. Wie wir um unsere Zukunft gebracht werden, München/Zürich 2007, gelten, der zahlreiche Schandtaten aufzählt, die seiner Meinung nach auf das Konto der 68er gehen). 
Nur gut ein Jahrzehnt nach der politischen Neuorientierung bildete sich in Deutschland, aber auch in anderen Staaten, ein neuer Ursprungsmythos heraus. Dem politischen Neubeginn nach 1945 musste nunmehr - gemäß dem von langer Hand vorbereiteten Programm der Frankfurter Schule - eine »intellektuelle Gründung « folgen. ${ }^{26}$ Deren Säulen sollten sein: Vergangenheitsbewältigung, Westbindung und Demokratisierung. Dass 1968 « in mancher seiner zahllosen Facetten nicht von diesem Mythos getrennt werden kann - das haben verschiedene Untersuchungen der letzten Jahre herausgearbeitet. ${ }^{27}$ Es können im vorliegenden Zusammenhang nicht die Hintergründe des »Mythos '68 « geklärt werden. ${ }^{28}$ Nur so viel sei gesagt: Liest man die inzwischen reichhaltige Erinnerungs- und Rechtfertigungsliteratur der einstigen Protagonisten ${ }^{29}$, so stößt man immer wieder auf gründungsmythische Floskeln, die auf die Behauptung hinauslaufen: Vor uns gab es nichts dergleichen! Wir sind die eigentlichen Gründer der Republik! $!^{30}$ Historisierung kann angesichts einer solchen, weit verbreiteten Deutung mythenzertrümmernd wirken.

In den folgenden Ausführungen sollen dafür einige Beispiele gegeben werden. Ein Stichwort lautet: Vergangenheitsbewältigung. Dieser Punkt war im Generationenkonflikt der 1960er-Jahre entscheidend. Häufig wurde die Auflehnung gegen die Väter zum Politikum, wobei auch private Hintergründe (der Konflikt mit dem eigenen Vater) hier und da eine Rolle spielten. Die 68er-Bewegung griff, ob bewusst oder nicht, Vorgaben auf, die den Vorstellungen der reeducation entsprachen. Diese

26 Ausführlich dargelegt bei Albrecht / Behrmann / Bock, / Homann / Tenbruck, Die intellektuelle Gründung der Bundesrepublik. Eine Wirkungsgeschichte der Frankfurter Schule, aaO. (FN 23). Die Studien setzen der wertend-kritischen Theorie der Frankfurter Schule und ihrer Erben die wertfrei-weberianische Methode entgegen; was in diesem Band als »Gründung « bezeichnet wird, ist für andere Historiker eine »Umgründung « (Manfred Görtemaker, Geschichte der Bundesrepublik. Von der Gründung bis zur Gegenwart, München 1999, S. 475-596); im Vergleich dazu stärker polemisch: Rolf Kosiek, Die Frankfurter Schule und ibre zersetzenden Auswirkungen, 6. Auflage, Tübingen 2005. Die Abhandlung ist aus explizit »rechter « Sichtweise geschrieben.

27 Vgl. die unterschiedlichen Beiträge bei Ingrid Gilcher-Holtey, (Hg), 1968 - Vom Ereignis zum Gegenstand der Geschichtswissenschaft, Göttingen 1998, bes. S. 239-307 (»Macht und Mythos der 68er Bewegungen«).

28 Gerd Langguth, Mythos '68. Die Gewaltphilosophie von Rudi Dutschke - Ursache und Folge der Studentenbewegung, München 2001, reduziert den Mythos '68 weitgehend auf die Gewaltphilosophie Rudi Dutschkes, was freilich etwas zu kurz gegriffen erscheint.

29 Exemplarisch seien genannt: Peter Mosler, Was wir wollten, was wir wurden. Zengnisse der Studentenrevolte, Reinbek bei Hamburg 1988; Tobias Mündemann, Die 68er ... und was aus ibnen geworden ist, München 1988; Dieter Kunzelmann, Leisten Sie keinen Widerstand! Bilder aus meinem Leben, Berlin 1998; Negt, Achtundsechzig. Politische Intellektuelle und die Macht, aaO. (FN 18).

30 Vgl. etwa Mosler, Was wir wollten, was wir wurden. Zeugnisse der Studentenrevolte, $\mathrm{aaO}$. (FN 29), der die Einleitung seines Bandes in gänzlicher Unbescheidenheit überschreibt: »Die Geburtsstunde der Republik«; mit Recht spricht Edgar Wolfrum, Die geglückte Demokratie. Geschichte der Bundesrepublik Deutschland von ibren Anfängen bis zur Gegenwart, Stuttgart 2006, S. 269, davon, derartige Interpretationen verschafften den »Eindruck einer zweiten `Stunde Null « . 
Rezeption war weitgehend deckungsgleich mit den Konzeptionen der Kritischen Theorie ${ }^{31}$, wobei freilich deren Einfluss im konkreten Fall schwer zu ermessen ist. Bei vielen Äußerungen von expliziten 68ern zeigt sich immer wieder von neuem der Ursprungsmythos in punkto Vergangenheitsbewältigung. Gelegentlich zitiert wird ein Ausspruch der früheren Justizministerin Herta Däubler-Gmelin, es habe einen »Aufschrei« ihrer »Generation gegen die totale Ruhe, gegen die Nichtbeschäftigung mit der Vergangenheit « seitens der so genannten Vätergeneration gegeben. ${ }^{32}$ Gegen dieses vollmundig vorgetragene Diktum ist zweierlei einzuwenden: Betrachtet man die Jahrgänge der viel zitierten »Vätergeneration«, so kann man unschwer eruieren, dass viele Angehörige dieser Alterskohorte zwischen etwa 1905 und 1925 geboren wurden. ${ }^{33}$ Das Führungspersonal des »Dritten Reiches « in Politik, Kultur und Militär gehörte aber in vielen Fällen schon zur Großvätergeneration der 68er. Die so gescholtenen Väter hatten zumeist keine höhere Weisungskompetenz und gelangten über Leutnants- und Oberleutnantsränge selten hinaus. ${ }^{34}$ Noch aus einem weiteren Grund ist der Ausspruch der SPD-Politikerin zu hinterfragen: Das Gefühl, vor 1968 habe es keine kritische Auseinandersetzung mit der deutschen Vergangenheit in den dunklen Jahren gegeben, hängt wohl eher mit der geringen historischen Bildung vieler seinerzeitiger Protestler zusammen als mit der Realität. Auch der wenig kundige Laie kann eine Reihe hervorragender Werke nennen, die ihren Siegeszug lange vor der als ominös geltenden Jahreszahl angetreten haben. Zu erwähnen sind in spärlicher Auswahl - lediglich die Best- und Longseller über den »SS-Staat « (Eugen Kogon), das Tagebuch der Anne Frank, die von dem Berner Historiker Walter Hofer edierte und vor allem in Schulen weit verbreitete Quellensammlung über den NS-Staat oder Karl Jaspers' viel gelesenes Buch über die »Schuldfrage«, das sogar die Kollektivschuld thematisierte - ein damals wie heute zumeist abgelehntes Pauschalurteil, das gleichwohl heftiger war als viele kritische Einwände der 68er gegen die Zeitgenossen des »Dritten Reiches «. ${ }^{35}$ Gewiss ist zu konzedieren, dass die kriti-

31 Friedrich H. Tenbruck, »Von der verordneten Vergangenheitsbewältigung zur intellektuellen Gründung der Bundesrepublik. Die Rahmenbedingungen «, aaO. (FN 23), S. 7796; Schrenck-Notzing, Charakterwäsche. Die Re-education der Deutschen und ihre bleibenden Auswirkungen, aaO. (FN 14), S. 267-278, der die »Vergangenheitsbewältigung « vor 1968 beschreibt, dadurch aber die Kontinuitäten aufzeigen kann; Manfred Heinemann, (Hg.), Umerziehung und Wiederaufbau. Die Bildungspolitik der Besatzungmächte in Deutschland und Österreich. Veröffentlichungen der Historischen Kommission der Deutschen Gesellschaft für Erziehungswissenschaft, Bd. 5, Stuttgart 1981.

32 Deutscher Evangelischer Kirchentag Frankfurt 1987. Dokumente, herausgegeben im Auftrag des deutschen Evangelischen Kirchentages von Konrad von Bonin, Stuttgart 1987, S. 549.

33 Näheres zur Generationenzuordnungsproblematik vgl. Clemens Albrecht, »Die Frankfurter Schule in der Geschichte der Bundesrepublik «, aaO. (FN 23), S. 498-519.

34 Näheres zu diesem Einwand bei Hermann Lübbe, »1968. Zur deutschen Wirkungsgeschichte eines politromantischen Rückfalls" wieder abgedruckt in: ders., Politik nach der Aufklärung. Philosophische Aufsätze, München 2001, S. 129-149, hier S. 135-137.

35 Belege für die intensive, kritische Auseinandersetzung mit dem Nationalsozialismus in den frühen Jahren der Bundesrepublik, die das Medium Film einschloss (»Die Mörder sind unter uns«), liefern in extenso die Buchautoren Hellmut Brunn / Thomas Kirn, 
sche Beschäftigung mit der deutschen Vergangenheit seit den späten 1960er-Jahren deutlich zunahm. Das hat aber nur zum Teil mit dem Wirken der 68er zu tun. In stärkerem Ausmaß ist die zunehmende Verbreitung und Diversifizierung der Medien in den letzten Jahrzehnten zu nennen. So konstatiert man eine intensivere Auseinandersetzung mit dem Holocaust, seinen Ursachen und Folgen seit der gleichnamigen Fernsehserie 1979, die auch für die bis heute andauernde omnipräsente Bedeutung dieses Wortes im kulturell-politischen Diskurs mitverantwortlich ist. ${ }^{36}$ Wie beim Thema »1968 «, ist auch bei der Rezeption der Vergangenheitskritik die Veränderung der Medienwelt zu berücksichtigen. ${ }^{37}$

Zudem ist gegen den oben zitierten Aufschrei Folgendes einzuwenden: Obwohl Hermann Lübbes kontrovers diskutierte Aussage vom »kommunikativen Beschweigen « der NS-Zeit erst 1983 fiel $^{38}$, hätte man vorher schon wahrnehmen können, dass das nach 1945 in Teilen der Bevölkerung gepflegte Schweigen über die jüngste Vergangenheit durchaus auch produktive, zukunftsweisende Seiten besaß. Der Wiederaufbau in allen Bereichen der Gesellschaft bedurfte, zumindest in der

Rechtsanwälte. Linksanwälte, Frankfurt/M. 2004, S. 49-57, bes. S. 49-51, die mit Recht ein »Trauma Nationalsozialismus « bei den 68er erkennen. Mit Traumata verbindet der Psychologe aber eine Störung des seelischen Gleichgewichts. Es geht also nicht selten um einen Krankheitsbefund, weniger um rational vertretbare, nachvollziehbare Formen der Vergangenheitskritik. In Erinnerung zu rufen sind auch die Einwände namhafter Historiker, die ihre akademische Karriere nach dem Zweiten Weltkrieg begonnen haben, an der These, kritische Vergangenheitsbewältigung hätte erst mit den 68ern begonnen. Neben Buchautoren wie Hans Buchheim und Hermann Graml ist besonders zu erwähnen: Karl-Dietrich Bracher, "Zwischen Geschichts- und Politikwissenschaft. Interview mit Werner Link « in, Hartmut Lehmann / Otto G. Oexle, (Hg.), Erinnerungsstücke. Weg in die Vergangenheit. Rudolf Vierhaus zum 75. Geburtstag gewidmet, Wien/Köln/Weimar 1997, S. 34, der als Belege nicht zuletzt seine Standardwerke über das Ende der Weimarer Republik sowie über die nationalsozialistische Machtergreifung nennen kann, die beide lange vor 1968 erschienen.

36 Zur kollektiven Erinnerungsgeschichte des Holocausts in Amerika, die Parallelen zu der in Deutschland erkennen lässt, vgl. Peter Novick, Nach dem Holocaust. Der Umgang mit dem Massenmord, Stuttgart/München 2001.

37 Martin Klimke / Joachim Scharloth, (Hg.), 1968. Handbuch zur Kultur- und Mediengeschichte der Studentenbewegung, Stuttgart/Weimar 2007; dieser Sammelband bietet eine synthetische Darstellung vieler Einzelstudien zum Thema, von denen lediglich folgende zu erwähnen sind: Christina von Hodenberg, »Der Kampf um die Redaktionen. >1968 und der Wandel der westdeutschen Massenmedien « in: dies. (Hg.), Wo »1968« liegt. Reform und Revolte in der Geschichte der Bundesrepublik, Göttingen 2006, S. 139-163, hier S. 144, die die »Linksverschiebung « (Habermas) im massenmedialen Bereich deutlich auf den Punkt bringt; ausführlicher: Christina von Hodenberg, Konsens und Krise. Eine Geschichte der westdeutschen Medienöffentlichkeit 1945-1973, Wallstein 2006, S. $397 \mathrm{ff}$.

38 Vgl. Hermann Lübbe, »Der Nationalsozialismus im politischen Bewußtsein der Gegenwart « in: Martin Broszat u.a. (Hg.), Deutschlands Weg in die Diktatur. Internationale Konferenz zur nationalsozialistischen Machtübernahme im Reichstagsgebäude zu Berlin. Referate und Diskussionen. Ein Protokoll, Berlin 1983, S. 329-349; zur Wirkungsgeschichte dieser These im Abstand von fast 25 Jahren vgl. Hermann Lübbe, Vom Parteigenossen zum Bundesbürger. Über beschwiegene und historisierte Vergangenheiten, München 2007. 
frühen Zeit, des Zusammenwirkens aller Kräfte und Zeitgenossen - ein Unternehmen, das durch zu heftige Kritik gefährdet worden wäre. Es wirkten ja verschiedene Gruppen am Wiederaufbau mit: ehemalige Gegner des NS-Regimes wie auch einstige Apologeten, Täter (ob verurteilt oder nicht) und besonders die Masse der Mitläufer. Letztere konnten nicht einfach ersetzt werden, denn ein Großteil der Bevölkerung ist schlichtweg nicht austauschbar. Trotz und wegen der sehr unterschiedlichen Haltung einzelner Bevölkerungsgruppen gegenüber den Nationalsozialisten und ihrer Lehre musste nach dem totalen Zusammenbruch ein modus vivendi gefunden werden. ${ }^{39}$ Solche Zusammenhänge hätten auch für jene plausibel sein sollen und können, die sich an die frühen Jahre der Bundesrepublik nicht mehr erinnern konnten.

Abschließend ist noch ein weiteres Argument vorzubringen, das die Notwendigkeit einer Einordnung von $» 1968$ « in das Kontinuum der Historie belegen soll. Schon die Begriffs- und Wortgeschichte der vielfältigen Phänomene der späten 1960er-Jahre ist in mehrfacher Hinsicht bedenkenswert. Zeitgenossen sprachen überwiegend von der APO, deren aktiver Kern dem SDS entstammte. ${ }^{40}$ Auch Benennungen wie »Studentenbewegung « oder »Studentenrevolte bzw. -rebellion« waren oft zu hören. Sie zieren diverse Buchtitel aus der damaligen Zeit. ${ }^{41}$ Aus dem Abstand von vier Jahrzehnten kann man problemlos erkennen, dass mit den genannten Kennzeichnungen nur bestimmte Organisationen und Protestformen erfasst werden, die Anlass für Konflikte mit dem »Establishment « wurden. Zu nennen sind in erster Linie zeitgenössische politische Ziele der Demonstrierenden wie der Kampf gegen die traditionelle Strukturierung der Universitäten, die Kritik am Kapitalismus sowie die Thematisierung der angeblich unbewältigten deutschen Vergangenheit. ${ }^{42}$ Diese Themen wurden auf zahllosen Veranstaltungen und »Demos « behandelt. Unzählige Plakate und Flugblättern zeugen davon.

Doch im historischen Gedächtnis blieb häufig anderes erhalten. Diese Neuakzentuierungen brachten auch neue Bezeichnungen hervor. So konnte man in den

39 Eine solche Haltung lässt sich in den 1950er-Jahren in der Politik weithin erkennen. Die integrationspolitischen Absichten haben auf längere Sicht deutlich mehr positive als negative Aspekte mit sich gebracht und sollten - auch aus heutiger Perspektive - keinesfalls ausschließlich als tendenziöse »Vergangenheitspolitik (Norbert Frei) oder gar "zweite Schuld « (Ralph Giordano) abgetan werden. Ein Beispiel für einen derartigen Versuch der Eingliederung verschiedener Altlasten in die frühe Bundesrepublik ist das viel kritisierte, wohlwollende Urteil von Spitzenpolitikern wie Kurt Schumacher oder Konrad Adenauer über die Mitglieder der Waffen-SS, die beiden Parteivorsitzenden als »eine Art vierter Wehrmachtsteil« (Schumacher) galt (vgl. dazu die Hinweise bei Heinrich A. Winkler, Der lange Weg nach Westen, Bd. II: Deutsche Geschichte vom »Dritten Reich « bis zur Wiedervereinigung, 3. Auflage, München 2001, S. 169).

40 Jürgen Briem, Der SDS. Geschichte des bedeutendsten Studentenverbandes der BRD seit 1945, Frankfurt/M. 1976; stärker wissenschaftlich ausgerichtet: Willy Albrecht, Der Sozialistische Deutsche Studentenbund (SDS), Bonn 1994; Tillmann Fichter, Kleine Geschichte des SDS, 4., überarb. und erg. Auflage, Essen 2007.

41 Vgl. etwa Helmuth Thielicke, Kritik der studentischen Rebellion, Tübingen 1969.

$42 \mathrm{Zu}$ diesen politischen Zielen vgl. etwa Lothar Voigt, Aktivismus und moralischer Rigorismus. Die politische Romantik der 68er Studentenbewegung, Wiesbaden 1991, S. 23. 
1970er-Jahren, als die Ereignisse noch nicht lange zurücklagen, gelegentlich von den »67ern « lesen. Man versuchte, den Anfang der Geschehnisse begrifflich zu betonen. In der Tat begann die Revolte mit der Tötung des Studenten Benno Ohnesorg anlässlich des Schah-Besuchs im Sommer 1967. Die Zeit der persönlichen Aufarbeitung setzte langsam ein, wobei die Rechtfertigungsabsichten offenkundig waren. ${ }^{43}$ Um 1980 herum bildete sich der Begriff »68er«. Es ist nicht vollständig geklärt, warum und wie es zu dieser Etymologie kam. Nachvollziehbar ist die Erklärung ${ }^{44}$, die im Anschluss an die kulturellen und sozialen Umbruchstendenzen im Laufe der 1970er-Jahre entstandenen Bürgerbewegungen, von der Anti-Atomkraft- und Umwelt- bis zur Frauenbewegung, hätten sich von ihrem übermächtigen Schatten absetzen wollen, indem sie ihn als $\$ 68$-Bewegung « benannten. Das Kürzel bekam schnell identifikatorische Relevanz, im Positiven wie auch im Negativen. Es liegen indes auch andere Hinweise auf die betreffende Wortprägung vor. Das Etikett, von den Medien und zahllosen ehemaligen Aktivisten begierig aufgegriffen, führte letztlich zu einem neuen Selbstbewusstsein. Das plakative »Wir, die 68er! « nahm langsam Gestalt an. Für die Akzentverschiebung spielte auch das eine Rolle, was sich nach 1968 ereignete. Um 1980 herum hatte man nicht nur die Vorfälle in den späten 1960er-Jahren vor Augen; man konnte mittlerweile das gesamte »rote Jahrzehnt « von 1967-1977 überblicken. ${ }^{45}$ Die Neue Linke der 1970er-Jahre, die aus dem Zerfall von »APO « und »SDS « hervorging, brachte neben gewaltfreien Veränderungsvorschlägen und Reformvorhaben auch den Terrorismus hervor, der die ursprünglichen Ziele der Studentenbewegung und ihrer Vordenker zumindest partiell diskreditierte. ${ }^{46}$ So lag es vor dem Hintergrund derartiger Kontinuitäten nahe, mit der Bezeichnung »68er « einen Horizont anzudeuten, der weit über vergangene (und immer mehr als obsolet erkannte) politische Absichten hinausgeht. Die Wortprägung kam auch späteren Tendenzen der Forschung entgegen, die mit wachsendem chronologischen Abstand immer mehr mit »1968 « verband: ${ }^{47}$ Protestbewegung, Generationenkonflikt, Kulturrevolution, Renaissance der marxistischen Ideologie, Spontaneis-

43 Als eines der vielen Beispiele sei genannt: Mosler, Was wir wollten, was wir wurden. Zeugnisse der Studentenrevolte, aaO. (FN 29).

44 Vgl. die Aussage Kraushaars, Wie wir uns befreiten. Michael Naumann und Wolfgang Kraushaar im Gespräch über 1968, die Folgen und das Selbstverständnis der Republik, aaO. (FN 20), S. 32 f.

45 Gerd Koenen, Das rote Jahrzehnt. Unsere kleine deutsche Kulturrevolution 1967-1977, 3. Auflage, Frankfurt a.M. 2006.

46 Zum Zusammenhang vgl. den kursorischen Überblick aus aktuellem Anlass: Felix Dirsch, »1968< und RAF. Zur Nähe und Ferne zweier zeitgeschichtlicher Phänomene«, in: Gegengift. Zeitschrift für Politik und Kultur 18 (2007, Heft 12), S. 5-11; zum Zusammenhang von »1968 « und RAF auch Lübbe, Endstation Terror. Rückblick auf lange Märsche, aaO. (FN 19), der nur relativ kleine Kontingente der 68er in den Untergrund wandern sieht, diese seien jedoch sehr konsequent gewesen und hätten das detailgenau umgesetzt, was in Flugblätter häufig propagiert worden sei: den Einsatz von Gewalt gegen die vermeintlich »repressiven « Staatsorgane.

$47 \mathrm{Zu}$ dieser Diversifikation der Forschung über »1968« vgl. Wolfrum, Die geglückte Demokratie. Geschichte der Bundesrepublik Deutschland von ihren Anfängen bis zur Gegenwart, aaO. (FN 30), S. 269. 
mus und Subversivität, Vergangenheitsbewältigung, liberalisierte Sexualmoral, Voraussetzung für den »spin-off « der neuen sozialen Bewegungen, neue Legitimation für Gewalt und vieles andere. Die 68er-Bewegung war eben aus der späteren Sichtweise mehr als das, was APO und SDS intendierten oder ausführten. Der historische Blickwinkel ist eben nicht identisch mit den zeitgenössischen Eindrücken und Erlebnissen. An dieser Stelle ist Broszats Bemühen in Erinnerung zu rufen, »Gedächtnis in Geschichte « zu verwandeln - ein Vorhaben, das mit wachsendem Abstand von den betreffenden Ereignissen zum geschichtswissenschaftlichen Projekt wird.

So ist zusammenfassend festzuhalten: Relevante historische Ereignisse bedürfen nach über einer Generation der Historisierung. Dies gilt selbst für die äußerst destruktiven totalitären Richtungen. Die Historisierung von »1968 « dürfte weit weniger kontrovers verlaufen als diejenige des Nationalsozialismus, weil die »Singularitäts-Klippe« fehlt. Bei allen Unterschieden im Einzelnen gibt die methodische Debatte über die Tragfähigkeit und Grenzen einer Historisierung des Nationalsozialismus grundlegende Hinweise bezüglich einer Übertragung dieses Konzepts auf andere geschichtliche Epochen. Auch »1968« ist hochgradig moralisierungs- und mythenanfällig, wie einige der vielfältigen Folgenabschätzungsdebatten zeigen. Einer solchen Tendenz kann durch eine sachliche Historisierungsdiskussion entgegengewirkt werden. Seit den Erinnerungsfeierlichkeiten zum dreißigsten Jahrestag 1998 wird immer mehr ersichtlich, dass »1968« zum »Gegenstand der Geschichtswissenschaft « wird $^{48}$ - ein Trend, der in Zukunft zunehmen dürfte. Aktuell-politische Kontroversen über ehemalige, militant-steinewerfende K-Gruppenführer wird es in absehbarer Zeit kaum noch geben, da sich der betreffende Personenkreis dann längst auf gut dotierte Professorenstellen in Deutschland oder den USA zurückgezogen haben wird. Dort können die mittlerweile Älteren ein Stück weit »Selbsthistorisierung « (Hermann Lübbe) ihrer schon länger zurückliegenden Handlungsweisen betreiben. Doch eine Historisierung entzieht auch kurzschlüssiger konservativer 68er-Kritik den Boden. So war anlässlich der Erinnerung an den 25. Jahrestag der Vorfälle zu lesen, die Republik solle sich von »1968 « emanzipieren. ${ }^{49}$ Betrachtet man die kulturellen und zivilisatorischen Voraussetzungen und Zusammenhänge, so ist ersichtlich, dass auch am Ende der 1960er-Jahre keine Dämonen auftraten, die einfach wieder, nach kurzer Zeit, verschwinden. Vielmehr handelt es sich um Vorgänge, die mit fundamentalen Entwicklungen der modernen Industriegesellschaft zusammenhängen, also nicht nur politisch zu verorten sind. Dass solche Tendenzen von kulturhistorischem Rang von nationalen Eigenheiten überlagert wurden, liegt in der Logik umfassender Umbruchsprozesse.

48 So der Titel eines wichtigen, von Ingrid Gilcher-Holtey herausgegebenen Sammelbandes: 1968 - Vom Ereignis zum Gegenstand der Geschichtswissenschaft, aaO. (wie FN 27); die Aufsatzsammlung gibt einen guten Einblick in die internationale Aufarbeitung der Vorfälle.

49 Eckhard Fuhr, »Alles Achtundsechziger«, in: Frankfurter Allgemeine Zeitung vom 27. März 1993, S.1. 
»1968« wird trotz seiner kaum zu überschätzenden symbolischen Relevanz zu keiner »Vergangenheit « werden, »die nicht vergeht « (Ernst Nolte), sondern im Laufe der Zeit unaufhaltsam zum Gegenstand der Historisierung mutieren. Die Frage ist lediglich, wie eine solche am besten durchzuführen ist.

\section{Zwei Beispiele für frühe Historisierungsversuche von "1968" am Ende der 1960er-Jabre}

Oben wurde bereits erwähnt, dass eine echte, d.h. methodisch reflektierte, Historisierung einige Jahrzehnte Abstand von den zu historisierenden Ereignissen benötigt. ${ }^{50}$ Nur auf diese Weise ist eine Kontextualisierung in das Kontinuum der Zeitgeschichte möglich. Es kann somit gezeigt werden, welche Strömungen zu den betreffenden Vorfällen hinführten, also den Hintergrund für sie bildeten. Gleichfalls wird ersichtlich, welche Verbindungs- und Rezeptionslinien in die unmittelbare Gegenwart führen. So wird das derart Eingeordnete einer bloß oberflächlichen politischen Moralisierung sowie einer mythischen Überhöhung entzogen..$^{51}$ Überdies kommt es zu einer Plausibilisierung der betreffenden Handlungen.

Nun war bereits vor vier Jahrzehnten das Bedürfnis zu erkennen, die Wurzeln der »Neuen Linken« zu dekuvrieren, um im politisch-wissenschaftlichen Meinungskampf die jeweiligen Gegner zu entlarven. In diesem Zusammenhang versuchten seinerzeit liberale und konservative Kritiker von APO und SDS, deren historische Vorläufer in Erinnerung zu rufen. Auf diese Weise sollte belegt werden, dass die betreffenden - scheinbar neuartigen - Phänomene gar nicht so neu sind, sondern letztlich Varianten uralter linker Erscheinungen darstellten - so jedenfalls die Sicht maßgeblicher konservativer Gegner. Um eine echte Historisierung im methodologischen Sinn handelt es sich dabei aber nicht. Lediglich diejenigen geschichtlichen Strömungen und politisch-ideologischen Richtungen konnten (z.T. in polemischer Absicht) untersucht werden, die zeitlich gesehen vor 1967/68 einzuordnen sind und zumindest teilweise - Vorläufer der Aktivisten darstellten, was besonders zwei Studien herausstellten. ${ }^{52}$ Man kann bei solchen Versuchen von einer retrospektiven Histo-

50 Dass dabei einem zeitlichen Abstand von 40 Jahren eine besondere Bedeutung zukommt - davon berichtet nach Auskunft Assmanns, Das kulturelle Gedächtnis. Schrift, Erinnerung und politische Identität, aaO. (FN 10), S. 51, bereits das alttestamentliche Buch Deuteronomium.

$51 \mathrm{Zu}$ einer solchen Überhöhung neigen paradoxerweise nicht nur die Befürworter und Apologeten von »1968«, sondern auch deren Gegner und Kritiker. Letztere tendieren nicht selten dazu, alles Negative mit diesem Datum in Verbindung zu bringen, was die Taten der damaligen Aktivisten ob ihrer Leistungen nachträglich gewissermaßen »adelt«.

52 Erwin K. Scheuch (Hg.), Die Wiedertäufer der Woblstandsgesellschaft. Eine kritische Untersuchung der "Neuen Linken" und ibrer Dogmen, Köln o.J. (1968); zur Kritik dieses Sammelbandes aus der neueren Forschung vgl. Gilcher-Holtey, 1968 « Eine versäumte Kontroverse, aaO. (FN 12), S. 61-64; zur Einordnung des Sammelbandes in die Phalanx der 68er-Gegner vgl. Dirsch, Mit spitzer Feder gegen den Zeitgeist. Ausgewählte konservative Zeitschriften und ihre Kritik an kulturrevolutionären Tendenzen, aaO. (FN 14), S. 73; als weitere bedeutsame zeitgenössische Abhandlung sei genannt: Caspar von Schrenck-Notzing, Zukunftsmacher. Die neue Linke in Deutschland und ibre Herkunft, Stuttgart 1968. 
risierung sprechen, die die Herkunft der betreffenden politischen Phänomene in lange zurückliegenden geschichtlichen Prozessen verorten will und diese Erscheinungen als Ausläufer wirkmächtiger, transepochaler Entwicklungen begreift. Eine echte Historisierung, die auch die Folgen berücksichtigt, war 1968 natürlich noch nicht möglich.

Einer der beiden Bände, vom Kölner Soziologen Erwin K. Scheuch ediert, hebt die eigenartige Verbindung alter wie neuer Elemente bei der »Neuen Linken« hervor: ${ }^{53}$ Einerseits sprang das spezifische marxistische Verhältnis von Theorie und Praxis ins Auge, gepaart mit revolutionären Leidenschaften (»Wiedertäufer«), die aus uraltem gesinnungsethisch-altruistischen Antrieb erwuchsen. Andererseits fiel bereits damals der Aktivismus auf, die spontaneistische wie subversive Tatenvielfalt mittels moderner Medien zu transponieren, obwohl es Jahrzehnte dauerte, bis die »Studentenbewegung im Spannungsfeld von Kultur-Revolution und Medien-Evolution « umfassend dargestellt wurde ${ }^{54}$.

Liest man aus heutiger Perspektive die Beiträge des Sammelbandes über die modernen »Wiedertäufer «, so kann man aus dem zeitlichen Abstand heraus problemlos erkennen, welche Beschreibungen und Diagnosen haltbar sind und welche nicht. Die Autoren der Studie, zumeist liberale Kritiker der Studentenbewegung, gehörten der mittleren Generation an. Sie zählten überwiegend nicht mehr zur Kriegsgeneration, sondern erlebten die letzten Kriegsjahre entweder als junge Wehrmachtssoldaten oder als Flakhelfer. Vom Alter der Rebellierenden trennt sie nur in Ausnahmefällen eine volle Generation. Einige der Publizisten mutierten infolge der Vorfälle zu Konservativen und beteiligten sich am »Bund der Freiheit der Wissenschaften «, der unter führender Beteiligung von Persönlichkeiten wie Hans Maier, Hermann Lübbe und Erwin K. Scheuch gegründet wurde. ${ }^{55}$

Einige Feststellungen über die Protestbewegung sind auch vom Blickpunkt der gegenwärtigen Forschung keineswegs überholt. Im Gegenteil: Wenn Scheuch dem harten Kern der Neuen Linken säkular-heilsgeschichtliche Ideale zuschreibt, so antizipiert er wichtige Resultate der neueren Forschung ${ }^{56}$. In der Tat: Die Vertreter der »ewigen Linken « (Ernst Nolte) kamen, obwohl nicht selten atheistisch ausgerichtet, häufig mit religiöser Inbrunst daher, um ihr existentielles Anliegen, den Protest gegen die aufgrund der Ungleichheit des Eigentums bewirkten Ungerechtigkeiten, auf diese Weise mit gebührender Emphase vorzutragen. Dass die Protagonisten meist aus den besseren Schichten stammten und sich für die Schlechtergestellten einsetzten, ist gleichfalls nicht neu. Schon bei den führenden Münsteraner Wiedertäufern lässt sich das belegen. Der Unterschied liegt indes auf der Hand: In früheren Epo-

53 Vgl. das aussagekräftige Vorwort von Scheuch, aaO. (FN 52), S. 7-12.

54 Vgl. bes. Katrin Fahlenbach, »Protestinszenierungen: Die Studentenbewegung im Spannungsfeld von Kultur-Revolution und Medien-Evolution«, aaO. (FN 37), S. 11-21.

55 Wichtige Hintergründe finden sich in der Studie von Till Kinzel, »Der >Bund Freiheit der Wissenschaft< und die >Notgemeinschaft für eine freie Universität< im Widerstand gegen die Achtundsechziger «, aaO. (FN 14), S. 112-136.

56 Exemplarisch: Simon Kießling, Die antiautoritäre Revolte der 68er. Postindustrielle Konsumgesellschaft und säkulare Religionsgeschichte der Moderne, Köln 2006, S. 155283; Gottfried Küenzlen, Der neue Mensch. Eine Untersuchung zur säkularen Religionsgeschichte der Moderne, München 1994, S. 174-200. 
chen der Geschichte konnte die »ewige Linke Menschen verweisen, die wirklich nicht mehr als ihre Ketten zu verlieren hatten. Über zwei Jahrzehnte nach dem Ende des letzten Weltkrieges bedurfte es einer aufwendigen Argumentation, um zu belegen, warum es auch ohne massenhaft Ausgebeutete und Benachteiligte zu einer Revolution kommen musste.

Den Autoren des von Scheuch herausgegebenen Sammelbandes (Ulrich Lohmar, Manfred Hättich, Peter Christian Ludz, Wilhelm Hennis etc.) ist aber klar, dass die neuen eruptiven Phänomene nicht allein mit einem vergangenheitsorientierten Blick zu deuten sind. Vielmehr spielten sie sich vor dem Hintergrund der zahllosen Veränderungen der Nachkriegszeit ab, von denen der Massenwohlstand wohl den grundlegendsten Wandel (im Vergleich zum Alltagsleben ein oder zwei Generationen früher) bedeutete. Die »Mitteilung eines neuen Lebensgefühls « bestimmte sämtliche Aktionen. ${ }^{57}$

Im Gegensatz zu den mehrheitlich liberalen Autoren des Bandes »Die Wiedertäufer der Wohlstandsgesellschaft « ist der Verfasser einer anderen, bis heute inspirativen zeitgenössischen Untersuchung über APO und SDS mit dem Titel »Zukunftsmacher « als Altkonservativer zu bezeichnen: Caspar von Schrenck-Notzing entstammt einer bekannten Münchner Adelsfamilie. Er geht in seiner Absicht, die Hintergründe der Neuen Linken aufzudecken, weiter als die Autoren in Scheuchs Sammelband. Die Tradition der »Studentendemonstrationsbewegung « sei bis zu ihren Wurzeln in der Aufklärungszeit zurückzuverfolgen. ${ }^{58}$ Dem Publizisten und Privatgelehrten fiel auf, dass einer ganzen Reihe von Verlagen, die den neuen Entwicklungen stets wohlwollend gegenüberstanden, »Aufklärungs-Traditionspflege « am Herzen lag. Die wesentlichen Kontinuitäten waren unschwer zu erkennen. Progressive Vertreter der aufgeklärten Kritik legten die Grundlagen für das Auseinandertreten von Moral, Fortschritt, Kritik, Freiheit und Glück einerseits sowie Autorität, Vorurteil, Zwang und Herrschaft andererseits. ${ }^{59}$ Die Französische Revolution bedeutete insofern eine maßgebliche Wendemarke im frühneuzeitlichen Utopie-Diskurs, als nunmehr evident wurde, dass manche Postulate dieser politiktheoretischen Richtung nicht bloße Illusion waren, sondern umsetzbar. Die Kategorie »Zukunft « erhielt somit eine reale Dimension. Schrenck-Notzing zeigt auf, welche Relevanz die Verschmelzung von Philosophie und Geschichte bei den Vätern der studentischen Bewegung erhielt ganz in der Nachfolge von Marx und Hegel. Überdies werden Anknüpfungspunkte der »Neuaufklärer « der unmittelbaren Gegenwart an Vorläufer der »48er-Bewegung « und an jene vor und nach dem Ersten Weltkrieg belegt.

Wenngleich Schrenck-Notzing an einigen Stellen seiner Darstellung abschweift und gelegentlich einen zu feuilletonistischen Stil bevorzugt - die »Zukunftsmacher « sind dennoch unentbehrlich und heute noch heranzuziehen. Seine Einordnung der zeitgeschichtlichen Phänomene in das Kontinuum der Historie verdeutlicht eine wichtige kulturphilosophische Dialektik: nämlich diejenige zwischen dem Alten

57 Scheuch, Zur Einleitung, aaO. (FN 52), S. 7.

58 Schrenck-Notzing, Zukunftsmacher. Die neue Linke in Deutschland und ihre Herkunft, aaO. (FN 52), S. 36.

59 Vgl. ebd., S. 35. 
und dem Modernen. Jedes Moderne wird einmal alt, manches Alte (wieder) modern. Gerade die Neue Linke kann ihre alten Wurzeln nicht verbergen. Schon die personellen Kontinuitäten sind offenkundig: Herbert Marcuse, herausragender Vordenker der Neuen Linken, wirkte in jungen Jahren, um 1920 herum, im Umfeld der linksradikalen USPD, die später größtenteils in der KPD aufging.

\section{Ansätze einer kulturphilosophisch wie-theoretisch fundierten Historisierung von $» 1968$ «}

Oben erwähnte Wissenschaftler und Publizisten wie Scheuch und Schrenck-Notzing bemühten sich 1968 um eine retrospektive historische Kontextualisierung der (zumindest vordergründig) neuen Phänomene. Das gelang ihnen weitgehend. Allerdings konnten sie - wie gesagt - natürlich eine solche Einordnung nur bezüglich der Herkunft von SDS und APO vornehmen. Heute, vierzig Jahre später, ist der Zeit- und Kulturhistoriker in der Lage zu überlegen, wie die 68er-Bewegung zwischen ihren Vorläuferströmungen und der nach 1968 weitergehenden Entwicklung zu verorten ist.

Ein derartiges Unterfangen kann im vorhandenen Rahmen nur andeutungsweise und skizzenhaft erfolgen. Lediglich einige Denkanstöße gebende Prolegomena sind vorzutragen. In diesem Zusammenhang ist der Stand der Forschung im Hinblick auf die 68er-Bewegung zu berücksichtigen. »1968 « bedeutet heute, wie bereits erwähnt, sehr vieles. Vor diesem Hintergrund bietet sich ein breiter Interpretationsrahmen an, der nicht nur die Umbrüche in den »dynamischen Zeiten « der »langen 1960 er-Jahre « zu beachten hat ${ }^{60}$, sondern über den chronologischen Horizont dieses Jahrzehnts hinausgehen soll. Auf diese Weise kann auch der Generationenkonflikt in die Analyse einbezogen werden.

Da die mit eruptiven Vorfällen verbundenen Veränderungen alle hoch entwickelten Industriegesellschaften betrafen ${ }^{61}$, erscheint es legitim, den umfassenden Maßstab der Zivilisations- und Kulturgeschichte zu wählen. So rückt - trotz aller Ambivalenzen der Bewegung - ihr spezifisch »moderner« Hintergrund in den Mittelpunkt der Betrachtung. Alle Jugendbewegungen haben mit dem Phänomen verkürzter Zeiten zu tun. ${ }^{62}$ Die tradierten Verhaltensmuster, Gewohnheiten, Weis-

60 Axel Schildt u.a. (Hg.), Dynamische Zeiten. Die 60er Jabre in den beiden deutschen Gesellschaften, Göttingen 2000. Der Band ist grundlegend für eine fundierte Analyse der Wandlungsprozesse dieses Jahrzehnts, dessen wesentliche Impulse erst Mitte der 1970er-Jahre endeten, als vor allem die Ölkrise und das Bewusstsein von den »Grenzen des Wachstums « das Ende der Reformeuphorie einläutete.

61 Naheliegenderweise stellen deshalb die meisten neueren Publikationen zum Thema die internationalen Zusammenhänge der Bewegung in den Mittelpunkt ihrer Argumentation, vgl. etwa die Überblicksdarstellung von Ingrid Gilcher-Holtey, Die 68er Bewegung. Deutschland - Westeuropa - USA, München 2001.

62 Vgl. aus seinen vielen Publikationen zum Thema lediglich: Hermann Lübbe, Im Zug der Zeit. Verkürzter Aufenthalt in der Gegenwart, 3., um ein Nachwort erw. Auflage, Berlin u.a. 2003; zur Relation von Modernität und Beschleunigung aller Lebensvorgänge vgl. Peter Borscheid, Das Tempo-Virus. Eine Kulturgeschichte der Beschleunigung, Frankfurt a.M. 2004. 
heiten, Sitten etc. veralten immer schneller, d.h. ihre Relevanz schrumpft in immer kürzeren Zeitintervallen. Der Erfahrungshorizont von Alt und Jung driftet daher stetig auseinander. Folgerichtig durchzieht die Erscheinung der Jugendrebellion die europäische Geschichte spätestens seit der Französischen Revolution. Als wichtige Stationen seien im Folgenden Beispiele aus Deutschland genannt, die zumeist auch eine europäische Dimension offenbaren. Goethes »Werther « bewirkte schon im Vorfeld dieses welthistorischen Datums eine Gebärde des Protests und der Selbstdarstellung. Bereits der Dichter des zündenden Roman-Erfolgs deutete die bald nach der Veröffentlichung wie Pilze aus dem Boden sprießenden Imitationen, Moden und Nippes-Varianten wie folgt: In »herrlichen Friedenszeiten« sei bei der »müßigen Jugend «, vornehmlich der studierenden, ein » Mangel an Taten« zu erkennen. Mit diesem Zitat lassen sich auch einige Hintergründe der 68er-Bewegung erklären, deren Aktivisten Krieg und Mangel nur mehr vom Hörensagen kannten. Von den Burschenschaften über das »Junge Deutschland « kann man problemlos einen Bogen zu den beiden wirkmächtigsten Jugendbewegungen des 20. Jahrhunderts schlagen: ${ }^{63}$ derjenigen nach dem Ersten Weltkrieg, die ihre Ursprünge bereits vor 1914 hatte, und der »zweiten deutschen Jugendbewegung (Hermann Lübbe). Erstere griff die Welt der Väter als individualistisch, materialistisch und naturfern an. Ihre Erfahrungen unterschieden sich von denen der Väter durch das »Stahlgewitter von 1914 « (Ernst Jünger). Die Grundwerte der nach 1890 Geborenen waren in starkem Maß geprägt durch Heimat, Natur, Familie und Kultur. Man kann also von überwiegend konservativen Tendenzen sprechen. Dagegen kamen jene Generationenauseinandersetzungen, die in den 1960er-Jahren kulminierten, in Deutschland dadurch zustande, dass die aufgrund der viel zitierten »Gnade der späten Geburt« zu Hause Gebliebenen gegen diejenigen zu Felde zogen, die noch mit persönlichen Erfahrungen über Krieg, Leid und Not aufwarten konnten. In beiden Fällen war der Krieg die große Wasserscheide zwischen den Generationen. Er wirkte als Katalysator der durch vollständig unterschiedliche Erlebnisse entstandenen Konflikte.

Die Veränderung der Lebenssituation breiterer Bevölkerungsschichten nach dem Zweiten Weltkrieg brachte einen Wertewandel sondergleichen mit sich, der als zivilisationsgeschichtlich fundierter Hintergrund der Unruhen berücksichtigt werden muss. Schon in den 1960er-Jahren existierte eine größere Zahl von Studien, die den Übergang von der industriellen zur technologisch-elektronischen Gesellschaft, heute meist als »Wissensgesellschaft « beschrieben, diagnostizierten und als unmittelbar bevorstehend herausarbeiteten. ${ }^{64}$ Die traditionellen Eliten wurden durch die neue

63 Zu den radikalen Burschenschaftlern als Vorreiter der Neuen Linken vgl. die historische Einordnung bei Schrenck-Notzing, Zukunftsmacher. Die neue Linke in Deutschland und ihre Herkunft, aaO. (FN 52), S. $41 \mathrm{ff}$.

64 Exemplarisch: Zbigniew Brzezinski, »Revolution oder Konterrevolution - zum historischen Standort des Revolutionarismus der >Neuen Linken «, aaO. (FN 52), S. 217-222, hier S. 217. Die Veränderungen werden heute meist als postmoderner oder postindustrieller Wertewandel beschrieben, der auch in der neuesten Forschung mit den 68ern in Verbindung gebracht wird (Kießling, Die antiautoritäre Revolte der 68er. Postindustrielle Konsumgesellschaft und säkulare Religionsgeschichte der Moderne, aaO. [FN 56]). 
Situation vielfach unsicher und neigten deshalb oft $\mathrm{zu}$ »Konzessionismus « - eine Einschätzung, die in gehörigem Abstand von einigen Jahrzehnten noch deutlicher auffällt. ${ }^{65}$ Die Neuerer nutzten ein solches Verhalten als Schwäche und präsentierten sich als selbstbewusste Revolutionäre. Dies galt um so mehr, da nicht wenige aus der angegriffenen Vätergeneration in Schuld verstrickt waren, was dem Engagement der »kritischen Jugend « noch zusätzliche Sympathien in der Öffentlichkeit einbrachte.

Allerdings kann die facettenreiche Thematik des Wertewandels die Hintergründe von »1968« nicht ausreichend erklären, insbesondere nicht die spontan-revolutionären Aktionen, deren Vielfalt vom bürgerlichen Ungehorsam bis zur Bombenexplosion reichte. Erste Unruhen machten sich bereits in den frühen 1960 er-Jahren bemerkbar. Häufig lässt man die Vorgeschichte der studentischen Rebellion mit den Schwabinger Krawallen von 1962 beginnen, die mittlerweile ebenfalls detailliert aufgearbeitet sind ${ }^{66}$.

An dieser Stelle hat die Analyse tiefer anzusetzen. Die notwendigen, wenngleich allein nicht hinreichenden Voraussetzungen der 68er-Bewegung hängen eng mit kulturellen Veränderungen der Industriegesellschaft zusammen. Sie hat durch technische Rationalisierung und Mechanisierung neue Freiräume geschaffen. Die Arbeitszeit konnte tendenziell deutlich abgesenkt werden. Ein immer größerer Teil der Bevölkerung war und ist in der Lage, relativ lange Zeiten in Schul- und Universitätseinrichtungen zu verbringen. Es ist in der Forschung und unter Zeitzeugen kaum umstritten, dass fundamentale Universitätsreformen bereits vor 1968 stattfanden, was die Voraussetzungen für die zunehmende Akademisierung der Gesellschaft schuf, ohne welche es die Studentenbewegung nicht gegeben hätte. ${ }^{67}$ Die 68er sprangen auch hier auf einen Zug auf, der bereits längst in Bewegung war. Letztlich können auch die Reformen auf dem Bildungssektor, partiell zumindest, als Präsuppositionen und weniger als Ergebnis der Proteste betrachtet werden. Nie war eine Generation - gemessen an den Parametern Zeit, Geld und Lebensgestaltungsmöglichkeiten - freier als die zwischen etwa 1940 und 1950 Geborenen. Das gilt vor allem im Hinblick auf den mittlerweile erreichten Wohlstand, der manchem studentischen Demonstranten bereits zum Ekel wurde, und die Bildungschancen. Der Anstieg der Studentenzahlen in Deutschland und in der übrigen westlichen Welt belegt das eindrucksvoll. Weiterhin sind die durch den Wirtschaftsboom wohl einzigartigen Berufsmöglichkeiten zu nennen, von der selbst Universitätsabsolventen der späten 1970er- und der 1980er-Jahre meist nur träumen konnten.

Nun brachten aber solche Freiheiten nicht nur neue Möglichkeiten, sondern auch erhebliche Zwänge mit sich. In dieser zivilisationsgeschichtlich einzigartigen Situa-

65 So Lübbe, »Konzessionismus dient aber nie der Beschwichtigung, sondern er hat Selbstschädigung zur Konsequenz«, aaO. (FN 14), S. 37-63.

66 Vgl. Gerhard Fürmetz (Hg.), »Schwabinger Krawalle«. Proteste, Polizei und Öffentlichkeit zu Beginn der 60er Jahre, Essen 2006.

67 Hinweise bei Lübbe, »Konzessionismus dient aber nie der Beschwichtigung, sondern er hat Selbstschädigung zur Konsequenz«, aaO. (FN 14), S. 43 ff. 
tion, die auch von ehemaligen Aktivisten des Öfteren reflektiert wurde ${ }^{68}$, machten sich erkennbare orientierungspraktische Probleme bemerkbar. Die sich nunmehr bietenden Dispositionsgelegenheiten wollten erprobt sein. Das Einüben neuer Formen sozialer Interaktion unter modernen Freiheitsbedingungen stellte ein weites Feld dar und umfasste alle Bereiche des gesellschaftlichen Zusammenlebens. Zu erwähnen sind (in knapper Auswahl) der extensive Drogenkonsum, mannigfache Versuche, ein neues Verhältnis zwischen den Geschlechtern zu finden (Freie-Liebe-Experimente, Kommunenkultur etc.), die Rezeption neuer Verhaltensweisen des zivilen Ungehorsams und der westlichen Musikkultur, aber auch neue Wege in der Erziehung, die vom Kinderladen und der Kinderkrippe bis hin zu vielfältigen antiautoritären pädagogischen Praktiken und antibürgerlichen Vorstellungen in der Sexualaufklärung reichten.

Die nicht ausbleibenden $»$ Desorientierungswirkungen von Freiheitsgewinnen $\aleph^{69}$ plausibilisieren zumindest teilweise die eruptiven Ausbrüche, besonders den Spontaneismus und den omnipräsenten Nonkonformismus. Die neuen zeitdisponiblen Möglichkeiten wurden nicht selten in »Lebensführungsprobleme « umgesetzt. ${ }^{70}$ Das erklärt in nicht geringem $\mathrm{Maß}$ den Inflationismus sämtlicher Formen des Diskurses, was wiederum an das "große Gespräch « der Frühromantiker erinnert ${ }^{71}$. Diese Gruppe, die sich vor allem um die Gebrüder Schlegel und um die Zeitschrift »Athenäum « bildete, musste sich der neuen Situation nach der Französischen Revolution stellen, die viele Menschen aus herkömmlichen Strukturen der alten Feudalgesellschaft freigesetzt hatte. Da die Zahl der Intellektuellen damals aber relativ klein war, unterschied sich die Lage sehr von derjenigen über 150 Jahre später. Es ist jedoch kein Zufall, dass die Vordenker des »herrschaftsfreien Diskurses « um 1968 den »Strukturwandel der Öffentlichkeit« nicht zuletzt in der Literaten- und Salonkultur um 1800 festmachten ${ }^{72}$, als

68 Vgl. beispielsweise die Aussagen von Rainer Langhans, der mit Recht darauf hinwies, dass seine Generation die erste war, die sich kaum Gedanken über das materielle Überleben machen musste, das immer gesichert war. So konnte man einen »neuen Menschen « konzipieren, »der sich in seinem Leben nicht mehr primär um sein physisches Überleben kümmern muss « (Klimke / Scharloth, »Du musst den Dingen ihre eigene Melodie vorspielen und sie werden zu tanzen beginnen<. Ein Gespräch mit Rainer Langhans«, aaO. [FN 37], S. 311-316, hier S. 313).

69 Lübbe, "Konzessionismus dient aber nie der Beschwichtigung, sondern er hat Selbstschädigung zur Konsequenz«, aaO. (FN 14), S. 46.

70 Ebd., S. 54; zu den endlosen Debatten über »Lebensführungsprobleme « vgl. statt vieler anderer Belege die Aussage eines 68er-Protagonisten, Daniel Cohn-Bendit: »Man hat alles ausdiskutiert, alles war politisch, die Politik, das Leben, die Beziehung. Es war eine permanente Diskussion der realen und möglichen Probleme. Das war kräfteaufreibend und faszinierend zugleich « (Sybille Krause-Burger, Joschka Fischer. Der Marsch durch die Illusionen, Stuttgart 1997, S. 97, zitiert nach Kurt Sontheimer, So war Deutschland nie. Anmerkungen zur politischen Kultur der Bundesrepublik, München 1999, S. 101).

71 So, mit kritischer Stoßrichtung, Carl Schmitt, Politische Romantik, 3., unveränderte Auflage, Berlin 1968, S. 192.

72 Zur Relation von Salonkultur und Öffentlichkeit vgl. Jürgen Habermas, Strukturwandel der Öffentlichkeit. Untersuchung zu einer Kategorie der bürgerlichen Gesellschaft. Mit einem Vorwort zur Neuauflage 1990, 3. Auflage, Frankfurt a.M. 1993, bes. S. 89 ff., S. $101 \mathrm{ff}$ (Salonkultur und Öffentlichkeit); S. 177 f (Romantik und Öffentlichkeit). 
die intellektuelle Schicht gezwungen war, sich über neue Lebens- und Verhaltensweisen $\mathrm{zu}$ verständigen, die eine fundamentale Konsequenz der epochalen Zäsur von 1789 darstellten.

Auch die von manchen nicht für möglich gehaltene Renaissance des Marxismus ist vor dem Hintergrund eines allgemein für notwendig erachteten Experimentalismus in den späten 1960er-Jahren zu erklären. War man in den 1950er-Jahren noch von einer allgemeinen Entideologisierung in weiten Teilen der Gesellschaft überzeugt, so hatten die zuerst kleinen Zirkel der neuen Avantgarden ${ }^{73}$, insbesondere die sich formierende Neue Linke, immer mehr Erfolg bei ihren Bemühungen, die staatlichen Ordnungen der westlichen Welt mittels einer Neuaneignung der kommunistischen Klassiker nachhaltig zu hinterfragen. 1966 stellten Demoskopen bei Untersuchungen über die Ansichten von Studenten fest, dass Anzeichen vorlägen, die eine Beschreibung der angehenden Akademiker als größtenteils anpassungsbereit, unkritisch und politisch desinteressiert nahelegen. Noch konnten Helmut Schelskys Analysen zur (von ihm so genannten) »skeptischen Generation« aus dem Jahre 1957 Gültigkeit beanspruchen. Ein bald allgegenwärtiger Hang zur Fundamentalkritik und zum »Romantizismus « war kaum vorauszusehen.

Der mit allerlei Getöse einhergehende, protuberanzenhafte Umschwung lässt maßgebliche Gründe in den Versuchen erkennen, die »orientierungspraktischen Folgelasten politischer, sozialer und kultureller Emanzipation mit den Mitteln einer ideologischen Wiedertaufe « zu bewältigen. ${ }^{74}$ Die Bindung an eine Ideologie hat in aller Regel identitätsstabilisierende Konsequenzen. Unterwirft man sich Heilslehren, ist man von komplexen, lebenspraktisch relevanten Organisationsnotwendigkeiten wenigstens zum Teil befreit. Es ist klar, wie das einzelne Gruppenmitglied zu denken und zu handeln hat. Die unter modernen Freiheitsbedingungen immer unsicherer werdenden Lebensverhältnisse erfahren auf diese Weise einen Ausgleich durch neue ideologische Gewissheiten. In den späten 1960er-Jahren aufblühende Führerkulte, weithin sichtbar durch bei Demonstrationszügen herumgetragene Lenin-, Ho Chi Minh- oder Maobilder, schufen vor allem im akademischen Milieu jene Identifikations- und Orientierungsmöglichkeiten, die in der tendenziell ligaturenarmen modernen Welt ansonsten nirgendwo zu bekommen waren ${ }^{75}$. Der kompensatorische Charakter der ideologieinduzierten Sinnstiftung steht also außer Frage.

Die oben erwähnten Zusammenhänge sind auch auf der biographischen Ebene zu erörtern. So ist die nachhaltige Verschiedenheit der curricula vitae zwischen den Angehörigen der so genannten Flakhelfergeneration einerseits und den Jahrgängen,

73 Vgl. Gilcher-Holtey, Die 68er Bewegung. Deutschland - Westeuropa - USA, aaO. (FN 61), S. 11-24; Michael Schmidtke, Der Aufbruch der jungen Intelligenz. Die 68er Jahre in der Bundesrepublik und den USA (Campus Historische Studien, Bd. 34), Frankfurt a.M. u.a. 2003.

74 Lübbe, »Konzessionismus dient aber nie der Beschwichtigung, sondern er hat Selbstschädigung zur Konsequenz«, aaO. (FN 14), S. 47.

75 Zur engen Relation von Moderne und schwachen Gemeinschaftsbindungen vgl. Norbert Bolz, Das konsumistische Manifest, München 2002, S. 9 f. 
die etwa zwischen 1940 und 1950 geboren wurden, andererseits aufzuzeigen. Odo Marquard, später ein namhafter Philosoph aus der Schule Joachim Ritters, erblickte 1928 das Licht der Welt. ${ }^{76}$ Kaum ein Wort dürfte ihm - angesichts der Erlebnisse seiner Jugendzeit und frühen Erwachsenenjahre - fremder gewesen sein als der Ausdruck »Freiheit«. Während des Krieges musste er eine politische Internatsschule besuchen. Noch kurz vor der deutschen Kapitulation wirkte er als Flakhelfer und geriet in Gefangenschaft. Nach der Entlassung war eine Heimkehr in seine nunmehr unter polnischer Verwaltung stehende Vaterstadt Stolp nicht mehr möglich. Der junge Marquard konnte 1947 nicht einfach ein Studium aufnehmen, wie es Jahre später problemlos möglich gewesen wäre. Vielmehr war es unumgänglich, sich durch ein »Schippsemester « den Beginn einer musterhaften akademischen Karriere erst zu erarbeiten. Am Anfang stand der Bauarbeiter, erst danach kam der geistig Tätige. Die frühe Biographie des nachmaligen Philosophieprofessors belegt beispielhaft, dass kaum Möglichkeiten einer freien, selbstbestimmten Organisation des eigenen Lebens seinerzeit vorhanden waren. Für ihn galt das ebenso wie für Millionen Angehörige seiner Generation.

Das änderte sich spürbar in den frühen 1960er-Jahren. Viele Zeitgenossen haben die Wandlungen, die um 1960 herum einsetzten, drastisch beschrieben. ${ }^{77}$ Mit dem zunehmenden Massenwohlstand breiteten sich auch bis dahin ungeahnte Dispositionsgelegenheiten aus. Die Mobilität stieg deutlich an, die Kommunikationsmöglichkeiten erweiterten sich stark durch die Zunahme von Rundfunk- und Fernsehgeräten sowie Zeitungsabonnements. In dem Alter, in welchem die Angehörigen der Flakhelfergeneration und die noch Älteren häufig ihre Gestellungsbefehle erhielten, verbrachten nun nicht wenige der zwischen 1940 und 1950 Geborenen ihre Ferien fern von Deutschland und Europa. Traumstrände waren das Ziel vieler Reisenden.

Die Desorientierungswirkungen, die aus den erwähnten Notwendigkeiten resultieren, unter modernen Freiheitsbedingungen neue Formen des Zusammenlebens zu finden, waren entscheidend für das Kaleidoskop der Widersprüche, die viele Aktionen der 68er begleiteten. ${ }^{78}$ Die heftigen Gegner des angeblichen US-Imperialis-

76 Vgl. die kurzen autobiographischen Hinweise bei Odo Marquard, »Abschied vom Prinzipiellen. Auch eine autobiographische Einleitung «, in: ders., Zukunft braucht Herkunft. Philosophische Essays, Stuttgart 2003, S. 11-29, hier S. 13 f; zum Vergleich sind aus der Fülle der Memoirenliteratur - auch die viel beachteten Erinnerungsbände der beiden mit Marquard fast gleichaltrigen Günter Grass, Beim Häuten der Zwiebel, 2. Auflage, Göttingen 2006, und Fest, Ich nicht. Erinnerungen an eine Kindheit und Jugend, aaO. (FN 17), heranzuziehen.

77 Joachim Fest, Begegnungen. Über nabe und ferne Freunde, Reinbek b. Hamburg 2006, S. 351, der die Veränderungen vor allem an der Spiegel-Affäre festmacht.

78 Vgl. dazu den knappen Überblick bei Dirsch, »1968 « und RAF. Zur Nähe und Ferne zweier zeitgeschichtlicher Phänomene, aaO. (FN 46), S. 5-7; auch Winkler, Der lange Weg nach Westen, Bd. II: Deutsche Geschichte vom »Dritten Reich « bis zur Wiedervereinigung, aaO. (FN 39), S. 252, betrachtet die Wirkungen der 68er als oft ungewollt und widersprüchlich: So bekämpften sie den Pluralismus als Verschleierung gesellschaftlicher Verhältnisse und trieben ihn dennoch weiter voran. Weiterhin waren den erklärten »Phantasten « Reformen ein Gräuel, dennoch setzten sie eine Reformeuphorie großen Ausmaßes in Gang. 
mus und leidenschaftlichen Amerikakritiker waren in hohem Maß dafür verantwortlich, dass die Verwestlichung irreversibel wurde. ${ }^{79}$ Aber auch in der persönlichen Lebensführung sind Widersprüche leicht ausgemacht. Ausgerechnet jene, die sich besonders gern mit kollektivistisch-marxistischen Kostümen schmückten, offenbaren in toto einen individualistisch-hedonistischen wie permissiven Lebensstil, der erst in jüngster Zeit stärker erforscht wurde ${ }^{80}$. Er erweist sich im Nachhinein als zukunftsträchtig und bereitete die individualistischen Tendenzen innerhalb der westdeutschen Gesellschaft vor, die seit den 1980er-Jahren immer stärker zu beobachten waren. Um ein weiteres Beispiel zu nennen: An der vergangenheitskritischen Haltung der 68er kann kein Zweifel bestehen. Allerdings brachte die Renaissance der Ideologie auch im Bereich der Geschichtsdeutung Zweideutiges hervor. Auf der Basis der marxistischen Faschismus-Doktrin wurde der Nationalsozialismus mitunter verharmlost, insofern man dessen prinzipielle Unterschiede zu demokratisch-marktwirtschaftlichen Systemen mehr oder weniger leugnete. Kapitalistisch waren angeblich beide: der Faschismus und die freiheitlich-demokratische Grundordnung - eine Gemeinsamkeit, die für eine unterschiedslose Negation bei-

79 Vgl. zur »westernization" der 68er Axel Schildt, "Vom christlichen Abendland zum modernen Pluralismus - eine Skizze der ideologischen Landschaft der fünfziger und sechziger Jahre«, in: ders., Ankunft im Westen. Ein Essay zur Erfolgsgeschichte der Bundesrepublik, Frankfurt a.M. 1999, S. 178; Winkler, Der lange Weg nach Westen, Bd. II: Deutsche Geschichte vom »Dritten Reich « bis zur Wiedervereinigung, aaO. (FN 39), S. 252, betont, dass die 68er zahlreiche Protestformen aus den USA übernommen hätten, etwa »Sit-in« oder »Go-in«; Manfred von Doering-Manteuffel, Wie westlich sind die Deutschen? Amerikanisierung und Westernisierung im 20. Jabrbundert, Göttingen 1999, S. 43, betont dagegen ebenso wie Eckhart Jesse, »1968 - und 25 Jahre später « in: Mut. Forum für Kultur, Politik und Geschichte, Nr. 315 (November 1993), S. 16, stärker als Schildt den ambivalenten Charakter der 68er gegenüber der westlichen Wertewelt; Bernd Ulrich, »Die deutsche Linke und der Westen «, in: Rainer Zitelmann / Karlheinz Weißmann / Michael Großheim, (Hg.), Westbindung. Chancen und Risiken für Deutschland, Frankfurt a.M./Berlin 1993, S. 243-258, hier S. 246, bringt die Zweideutigkeiten der Linken gegenüber der westlichen Kultur auf die paradox anmutende Formel, dass die Linke ihre "anti-westliche Politik ins Extrem getrieben « habe, weil »sie verwestlicht war «; öfters will man das Dilemma dadurch auflösen, dass man bei vielen 68ern zwischen der Ablehnung des westlichen politischen Systems und der grundsätzlichen Affirmation westlich-kultureller Werte unterscheidet (vgl. dazu die Hinweise bei Sontheimer, So war Deutschland nie. Anmerkungen zur politischen Kultur der Bundesrepublik, aaO. [FN 70], S. 106, der das Werk der 68er, vor allem ihre Antinomien, nicht als Erfolgsstory begreift).

80 Vgl. zu den hedonistischen Selbstverwirklichungspostulaten in Kommunen- und Subkultur der 68er Joachim Scharloth, »Die Sprache der Revolte. Linke Wörter und avantgardistische Kommunikationsstile«, aaO. (FN 37), S. 223-234, hier S. 230-232, der vor allem deren Kommunikationsstil in den Mittelpunkt seiner Argumentation rückt. Andere Kenner der zeitgeschichtlichen Hintergründe wie Ingrid Gilcher-Holtey heben die Vermengung kollektivistischer wie individualistischer Elemente innerhalb der 68erBewegung hervor. Die Sozialhistorikerin betont aber, dass im Resultat »das politische Programm mehr und mehr dem Kult individueller Betroffenheit wich « (zitiert nach Winkler, Der lange Weg nach Westen, Bd. II: Deutsche Geschichte vom »Dritten Reich« bis zur Wiedervereinigung, aaO. [FN 39], S. 253). 
der so stark differierender Herrschaftsformen ausreichte. Selbst gegenüber Judentum und Zionismus legte die 68er-Bewegung ein zwiespältiges Verhältnis an den Tag. Zwar war die Linke vor 1967 insgesamt prosemitisch eingestellt, nach dem Sechstagekrieg Israels änderte sich das jedoch. ${ }^{81} \mathrm{Ab}$ diesem Zeitpunkt überwog der Antizionismus. Während sich eine Reihe von 68er-Protagonisten als Juden stilisierte $^{82}$, waren die Ausfälle gegen jüdische Professoren wie Kurt von Fritz, Ernst Fraenkel, Helmut Kuhn oder Richard Löwenthal auf diversen universitären Veranstaltungen für liberal-konservative Wissenschaftler ein wichtiger Grund, entschieden gegen die Militanz der Neuen Linken zu opponieren ${ }^{83}$. Auf eine Vielzahl weiterer Widersprüche, etwa im Organisationsaufbau und in der Propaganda der Protestler, kann an dieser Stelle nicht eingegangen werden. Eindeutig aber ist: Die merkwürdige Kombination »aus alten Ideen und brandneuen Ausdrucksformen« verblüffte nicht wenige Zeitgenossen. ${ }^{84}$ Sie ist vor dem Hintergrund der spezifischen kulturund zivilisationsgeschichtlichen Situation, in der die 68er-Bewegung agierte, zu erklären.

Zusammenfassend ist festzustellen: Eine Historisierung des Wirkens der 68er ist hilfreich, um lange gehegte Legenden zu entmystifizieren. Einer rein aktuell-tagespolitischen Instrumentalisierung (abhängig vom politischen Standpunkt) kann auf diese Weise methodisch-wissenschaftlich entgegengetreten werden. Angesichts der Vielfalt dessen, was in der gegenwärtigen Forschung als Tat und Erbe der 68er gilt, bietet sich eine Einordnung in das kulturhistorische Kontinuum an. Alle anderen Maßstäbe der Einbettung in größere historische Zusammenhänge haben sich zwar nicht als falsch, aber doch als zu kurz gegriffen erwiesen..$^{85}$ So wird evident, dass die

81 Vgl. Martin Klonke, »Das zionistische Staatsgebilde als Brückenkopf des Imperialismus<. Vor vierzig Jahren wurde die neue deutsche Linke antiisraelitisch « in: Merkur 61 (Juni 2007), S. 487-497.

82 Kießling, Die antiautoritäre Revolte der 68er. Postindustrielle Konsumgesellschaft und säkulare Religionsgeschichte der Moderne, aaO. (FN 56), S. 274-283.

83 Vgl. dazu Hans Maier, »Als Professor im Jahre 1968«, in: Schubert, 1968, aaO. (FN 19), S. 81-96, hier S. 88, zur Angst des Berliner Politologen Ernst Fraenkel vor einer »neuen SA « vgl. auch Wilhelm Bleek, Geschichte der Politikwissenschaft in Deutschland, München 2001, S. 369; die Diskussion über den Antizionismus, manche sprechen sogar von Formen des Antisemitismus, kam vor wenigen Jahren nach Kunzelmanns Geständnis, eine von ihm in einem jüdischen Gemeindehaus 1969 gelegte Bombe sei nicht explodiert, erneut in Gang; vgl. dazu Kraushaar, Die Bombe im Jüdischen Gemeindehaus, $\mathrm{aaO}$. (FN 15).

84 So Scheuch, Zur Einleitung, aaO. (wie FN 52), S. 7; ein interessierter Beobachter des Zeitgeschehens sah in den Erscheinungen von 1968 eine »explodierende Altertümlichkeit« (Thomas Mann) und betrachtete sie als erneuten Versuch, die Welt nach idealen Vorgaben zu gestalten, wobei eher Hegel als dessen Schüler Marx Pate stand (vgl. Hans Maier, "Geistige Umbrüche in Deutschland 1945-1995 in: Deutschland 1945-1995. Betrachtungen über Umbrüche, München 1995, S. 13-39, hier S. 20).

85 Häufig werden nur Teilaspekte der Studentenbewegung erfasst; vgl. als Beispiel die viel beachtete Studie von Rüdiger Safranski, Romantik. Eine deutsche Affäre, München 2007, S. 384-392, der die romantische Geistesströmung transepochal beschreibt und den schon von zeitgenössischen Kritikern festgestellten »Romantizismus (Joachim C. Fest) der 68er als Ausläufer einer langen Traditionslinie begreift. 
mittlerweile grau melierten Damen und Herren, 40 Jahre nach ihrem Auftritt auf der geschichtlichen Bühne, nicht selten weit überschätzt werden. Sie waren nur ein Element im Kontext weitreichender Umbrüche. Das Ergebnis einer konsequenten Historisierung deckt sich weitgehend mit Forschungsresultaten aus dem Bereich der Sozialhistorie, die hervorheben, dass Bewegungen aufgrund mangelnder Institutionalisierung lediglich fluiden Charakter aufweisen und so meist nur zur Mobilisierung dienen können. ${ }^{86}$ Ergo sind sie kaum in der Lage, zum Wandel sozialer Strukturen mehr als nur erste Anstöße zu liefern, da ihr Zerfall nur eine Frage der Zeit ist. Die Mißachtung dieser Erkenntnis ist hauptsächlich für das verantwortlich, was schon seit längerer Zeit als »Mythos '68 « durch die Medien geistert.

\section{Zusammenfassung}

40 Jahre nach den Ereignissen erscheint eine Historisierung von »1968« als angemessen und notwendig. Eine solche Debatte kann auf bestimmte Ergebnisse der Historisierungsdiskussion über die Zeit des Nationalsozialismus zurückgreifen. Eine konsequente Einordnung der 68er in das Kontinuum der Zeitgeschichte kann mythen- und legendenzertrümmernd wirken. Die seinerzeit Rebellierenden waren nicht an allem schuld. Eine »Neugründung der Republik«, wie von Freunden und Feinden der damaligen Aktivisten öfters vermutet, hat nicht stattgefunden. Weder setzte eine kritische Aufarbeitung der Vergangenheit erst in den späten 1960er-Jahren ein, noch ist das »kommunikative Beschweigen « der NS-Untaten in breiteren Bevölkerungsschichten der 1950er-Jahre ausschließlich negativ zu bewerten - ein Befund, der keinesfalls bedeutet, dass der Umgang mit dem »Dritten Reich « in der frühen Nachkriegszeit keine kritikwürdigen Seiten aufzuweisen hätte. Für eine Historisierung der Unruhen vor vier Jahrzehnten ist am besten der Maßstab der Kultur- und Zivilisationsgeschichte $\mathrm{zu}$ wählen, da die eruptiven Ausbrüche alle bedeutenden westlichen Industrienationen betrafen. Die modernen industriegesellschaftlichen Entwicklungen haben Freiheitsspielräume in hohem Maß geschaffen, etwa größere Zeitdispositionen und mehr Bildungsmöglichkeiten. Derartige neue Möglichkeiten mussten auf allen sozialen Sektoren erprobt werden. Als Folge sind erhebliche Desorientierungswirkungen auszumachen. Die ideologischen Bindungen boten den Aktivisten mehr oder weniger Halt und Gewissheit in einer tendenziell ligaturenarmen und unsicheren modernen Welt. Aufblühende Führerkulte der späten 1960er-Jahre sind daher als Kompensationen oder Pseudokompensationen dieser Desorientierungswirkungen zu begreifen.

86 Vgl. Gilcher-Holtey, Die 68er Berwegung. Deutschland-Westeuropa-USA, aaO. (FN 61), S. $111 \mathrm{f}$. 


\title{
Summary
}

Historization of $>1968$ < is necessary owing to the ageing of a generation, i.e. agegroups born around 1938-1948 who were responsible for the political actions. The events of those days more and more grow subject of the science of history. How should this contextualisation be embedded into the continuity of history? Obviously a more comprehensive point of view should be chosen. Never before a generation had comparable privileges. However a situation quite novel in the history of civilization (considering prosperity and higher education) needs to be tested, and also causes new constraints. This may explain some explosive accompanying circumstances of the revolt. Industrial rationalization brought forth additional time to be disposed in a new way - e.g. demonstrations, endless debates, teach-ins, sit-ins. In a retrospective glance, many phenomena seem to be created by a major group of activists but are plausible only by analyzing the overall modernization process. The $>68$ ers $<$ were only one decisive element - not more.

Felix Dirsch, Revolt of students or revolution of culture? The historization of the $» 68 \mathrm{ers}$ «

\section{Extremismus in Deutschland}

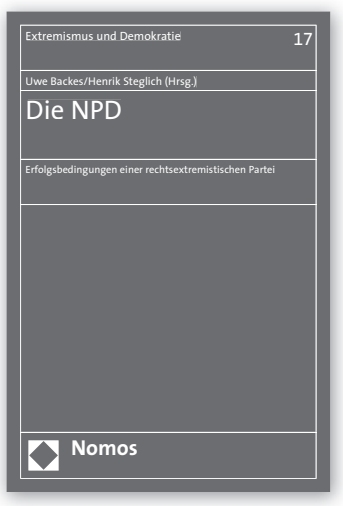

\begin{abstract}
Die NPD
Erfolgsbedingungen einer

rechtsextremistischen Partei

Herausgegeben von Uwe Backes und

Henrik Steglich

2007, 426 S., brosch., 49,- $€$, ISBN 978-3-8329-3122-3

(Extremismus und Demokratie, Bd. 17)

Die NPD konnte bei Wahlen wachsende Stimmengewinne verbuchen, in zwei Landesparlamente einziehen und sich besonders im östlichen Deutschland mancherorts sozial verankern. Ausgewiesene Experten analysieren die Erfolgsbedingungen der Partei, ein Who's who führender Parteirepräsentanten vervollständigt den Band.
\end{abstract}

Bitte bestellen Sie bei Ihrer Buchhandlung oder bei Nomos | Telefon 07221/2104-37 | Fax-43 | www.nomos.de|sabine.horn@nomos.de

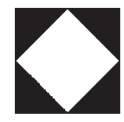

Nomos 